This document was prepared as an account of work sponsored by the United States Government. While this document is believed to contain correct information, neither the United States Government nor any agency thereof, nor The Regents of the University of California, nor any of their employees, makes any warranty, express or implied, or assumes any legal responsibility for the accuracy, completeness, or usefulness of any information, apparatus, product, or process disclosed, or represents that its use would not infringe privately owned rights. Reference herein to any specific commercial product, process, or service by its trade name, trademark, manufacturer, or otherwise, does not necessarily constitute or imply its endorsement, recommendation, or favoring by the United States Government or any agency thereof, or The Regents of the University of California. The views and opinions of authors expressed herein do not necessarily state or reflect those of the United States Government or any agency thereof or The Regents of the University of California. 
LBNL-, NDCXII-, HIFAN-

\title{
Capacitive Beam Position Monitor for NDCX-II*
}

\author{
Steve Lidia ${ }^{\dagger}$ and Frank Bieniosek, Enrique Henestroza \\ Accelerator and Fusion Research Division, \\ Lawrence Berkeley National Laboratory \\ Jeffrey Takakuwa \\ Engineering Division, Lawrence Berkeley National Laboratory
}

\begin{abstract}
This note describes the design, fabrication and calibration of the NDCX-II capacitive beam position monitor.
\end{abstract}

\footnotetext{
${ }^{*}$ This work was supported by the Director, Office of Science, Office of Fusion Energy Sciences, of the U.S. Department of Energy under Contract No. DE-AC02-0うCH11231.

†Electronic address: smlidia@1bl.gov
} 


\section{INTRODUCTION}

\section{NDCX-II Beamline}

The NDCX-II accelerator beamline is shown in Figure 1. The initial installation of the accelerator facility consists of an injector followed by 27 cells, a neutralized drift section and, finally, a target chamber or beam dump. The accelerator cells are categorized into 12 active acceleration modules, 9 inactive acceleration modules, and 6 diagnostic cells. This is shown in Figure 2 All cells are equipped with pulsed solenoid magnets for beam transport. A capacitive beam position monitor (BPM) is placed in each diagnostic cell, with a final BPM sited between the final active accelerator cell and the entrance to the neutralized drift section.

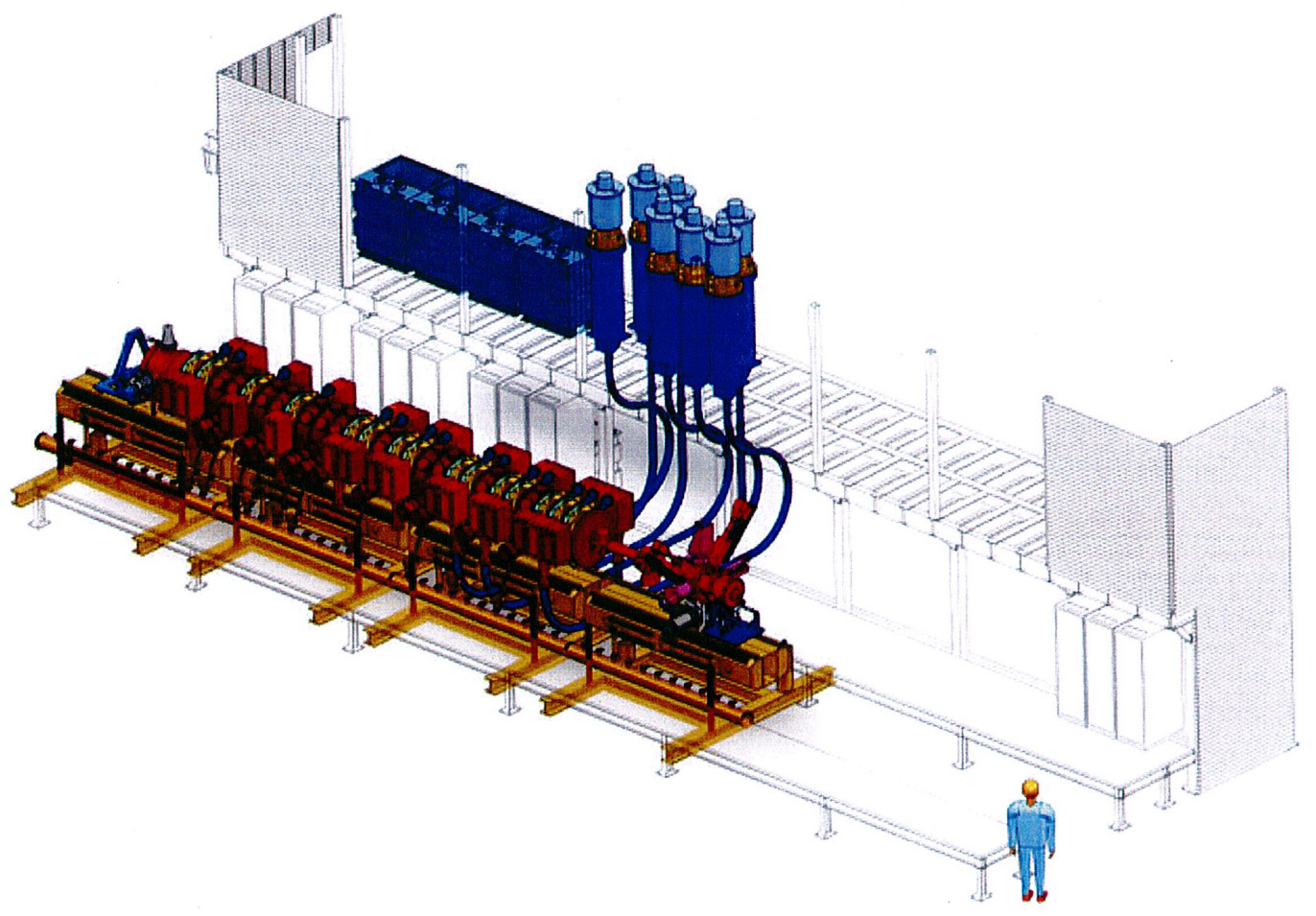

Figure 1: NDCX-II beamline. 


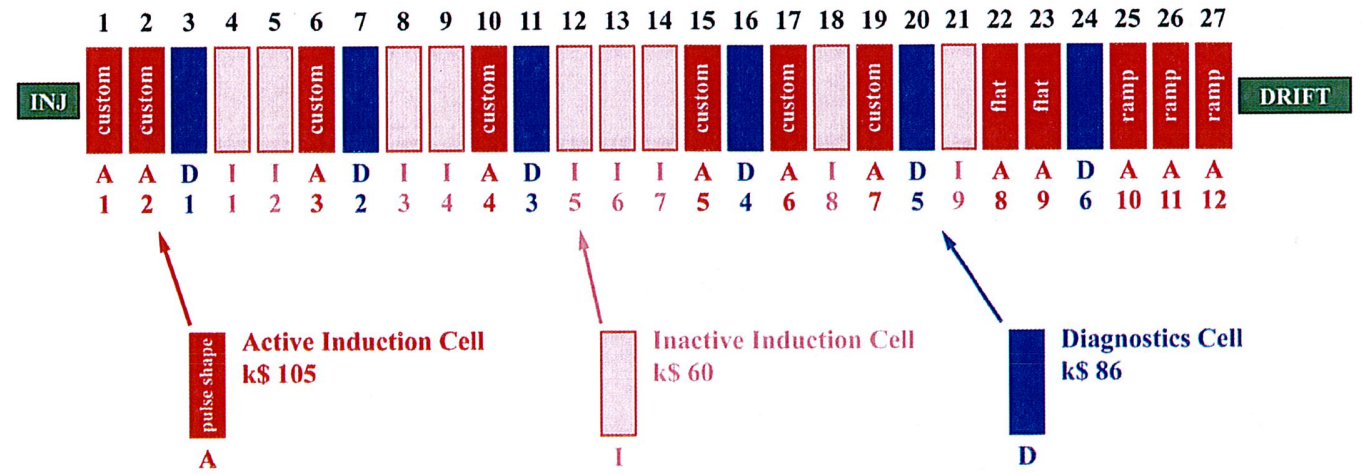

Figure 2: Beamline schematic

\section{Diagnostic Cell}

The NDCX-II diagnostic cell is shown in Figure 3. Each diagnostic cell is equipped with a capacitive beam position monitor (BPM), a pulsed solenoid, and a pair of DC dipole corrector magnets coaxial to the solenoid. The ability to retract the solenoid will enable the insertion of intercepting diagnostics. This functionality will appear only after commissioning and retrofit of the diagnostic cells.

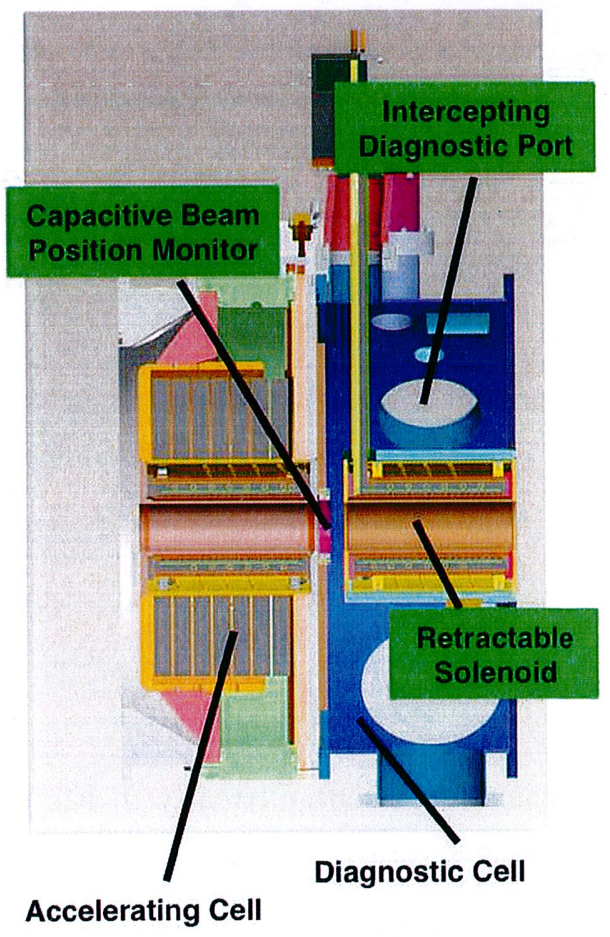

Figure 3: Diagnostic cell view 


\section{BASIC DESIGN}

The capacitive BPM is an axially short (compared to the lattice period and solenoid length) device that responds to the electric field of a passing charge distribution. We have chosen to use a standard design based around four isolated electrodes that cover the entire $2 \pi$ of azimuth [1]. This device provides measures of the total beam line charge density as well as horizontal and vertical position offset.

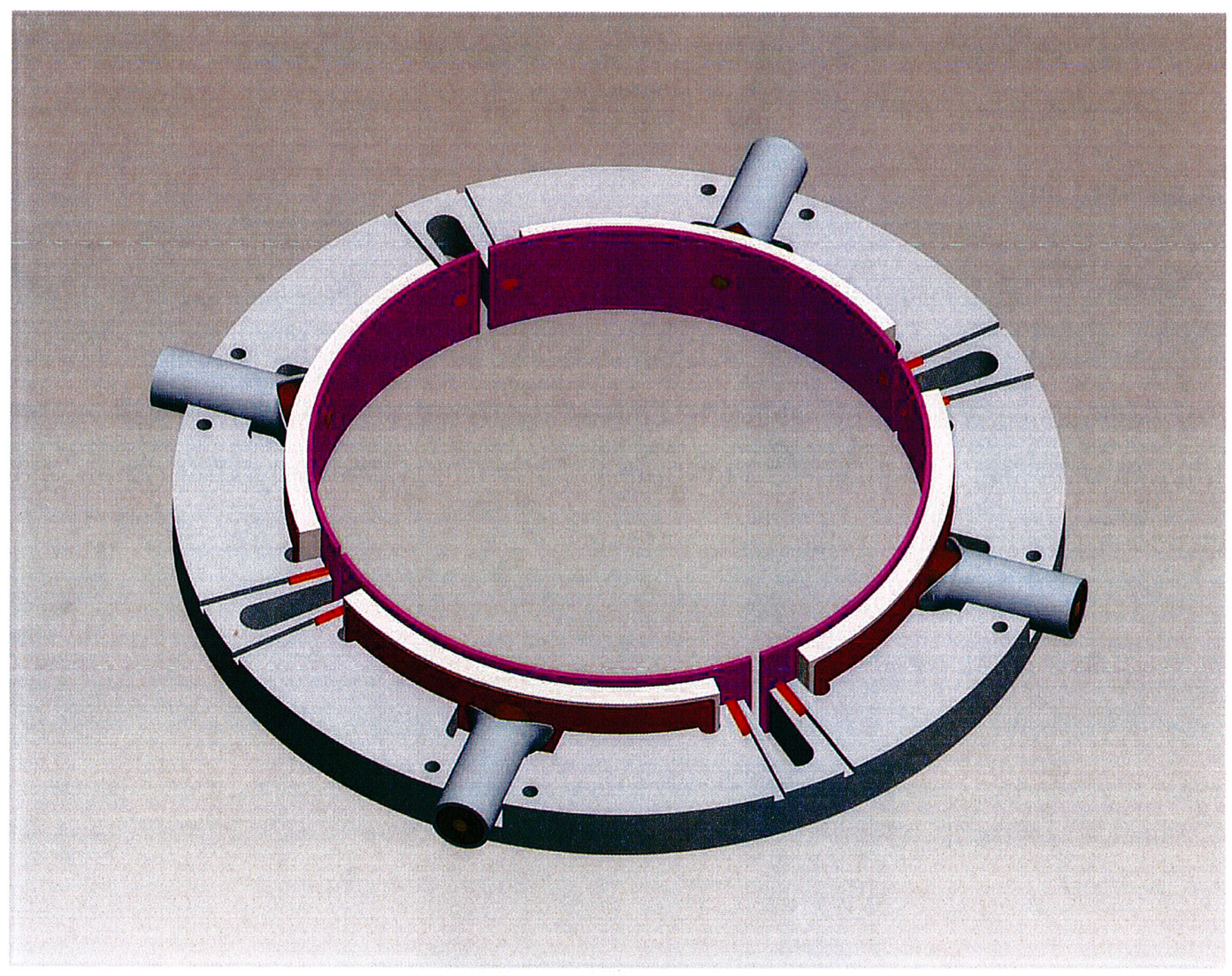

Figure 4: BPM schematic

\section{A. Geometry and materials}

The interior view of the BPM is shown schematically in Figure 4. The dimensions of the BPM are designed to match the NDCX-II beam tube which has an 3.125 -inch $(\sim 8 \mathrm{~cm})$ inner diameter. The 90-degree sector electrodes have a 4.17-cm radius, and are manufactured from OFE copper. The PEEK insulator has a $1 \mathrm{~mm}$ thickness and a dielectric constant of 


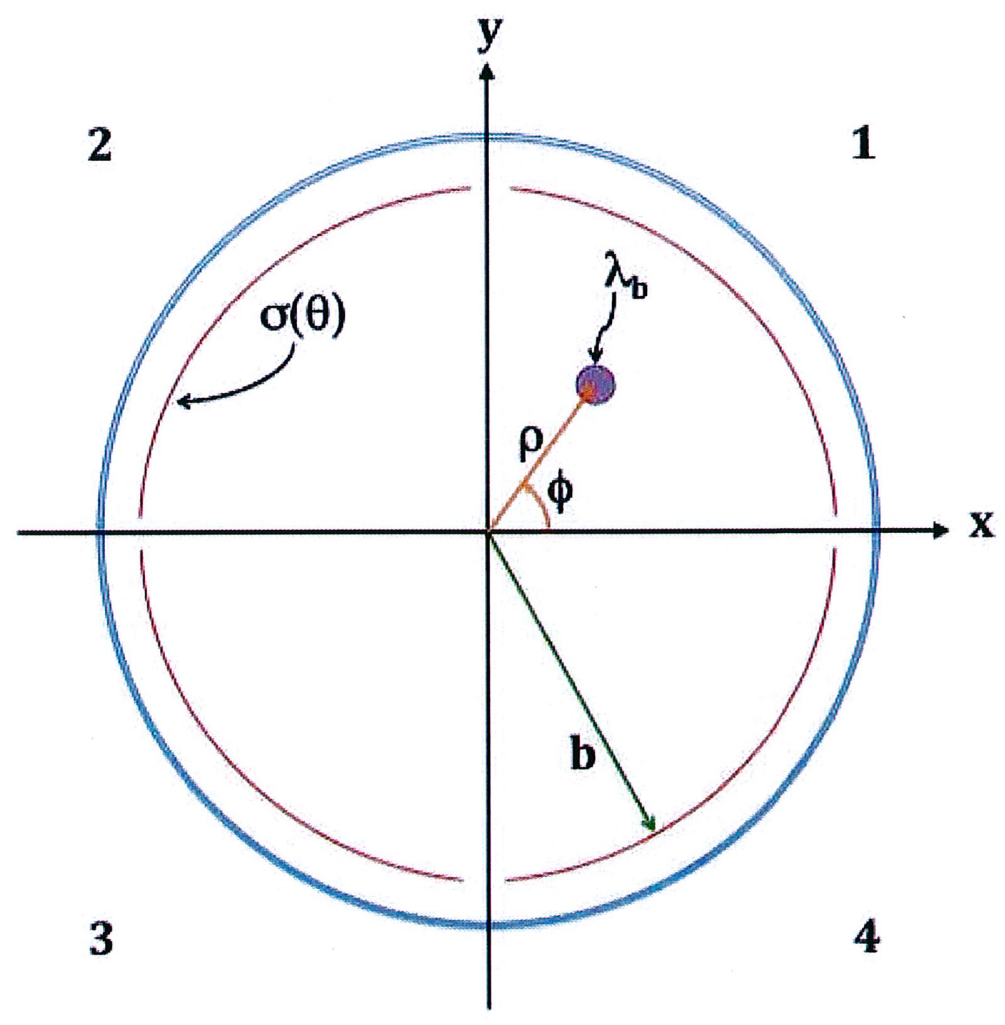

Figure 5: BPM geometry and charge configuration

3.3 at $1 \mathrm{MHz}$. The ground plate is also OFE copper, and is soldered to the ground shield of the 50- $\Omega$, RG-214 coaxial cable. The center conductor penetrates (with isolation) the ground plate and insulator and is soldered to the inner electrode plate. The outer housing and support structure is also manufactured from PEEK. The electrode axial length is 1.27 $\mathrm{cm}(0.5 \mathrm{inch})$. Each electrode analyzer branch circuit is constructed as a small capacitance to ground in parallel with the $50-\Omega$ line impedance.

\section{B. Beam model and surface charge configuration}

A simplified model of the BPM and beam is shown in Figure 5 . The beam itself is modeled as a quasi-static line charge density, $\lambda(t)$, with planar polar coordinates $(\rho, \theta)$. The pickup electrodes are sectors at a radius, $b$, and subtend nearly $\Delta \phi=\pi / 2$ radians (the difference is negligible for this discussion, but can be calibrated). They intercept fields from the moving charge over a length $L$. We assume, from an electrostatics perspective, that the 
outer conductor is maintained at electrical ground potential so that the net enclosed charge is zero. The induced surface charge on the electrodes will then have opposite polarity to that of the ion beam. The induced surface charge density is found to be [1]:

$$
\sigma(\theta)=\frac{-\lambda_{b}}{2 \pi b}\left[\frac{b^{2}-\rho^{2}}{b^{2}+\rho^{2}-2 b \rho \cos (\theta-\phi)}\right]
$$

or

$$
\sigma(\theta)=\frac{-\lambda_{b}}{2 \pi b}\left[1+2 \sum_{n=1}^{\infty}\left(\frac{\rho}{b}\right)^{n} \cos (n\{\theta-\phi\})\right] .
$$

The first expression is obtained from an image charge representation, while the second results from a multipole sum analysis. We will use either expression as is convenient to the analysis. The charge accumulated on the electrodes can be calculated by straightforward integration of Eqn. 1 or 2. We proceed by labeling the sectors in the usual way $(1,2,3,4$ counterclockwise - see Figure j), thus

$$
\begin{aligned}
Q_{\text {plate }}=L b \int_{\text {sector }} d \theta \sigma(\theta) \\
Q_{1}=\frac{-Q_{b}}{2 \pi} \int_{0}^{\pi / 2} d \theta \frac{1-\bar{\rho}^{2}}{1+\bar{\rho}^{2}-2 \bar{\rho} \cos (\theta-\phi)} \\
=\frac{-Q_{b}}{2 \pi} \int_{0}^{\pi / 2} d \theta\left[1+2 \sum_{n=1}^{\infty} \bar{\rho}^{n} \cos (n\{\theta-\phi\})\right], \\
\left.Q_{2}=\frac{-Q_{b}}{2 \pi} \int_{\pi / 2}^{\pi} d \theta \frac{1-\bar{\rho}^{2}}{1+\bar{\rho}^{2}-2 \bar{\rho} \cos (\theta-\phi)}\right] \\
=\frac{-Q_{b}}{2 \pi} \int_{\pi / 2}^{\pi} d \theta\left[1+2 \sum_{n=1}^{\infty} \bar{\rho}^{n} \cos (n\{\theta-\phi\})\right], \\
\left.Q_{3}=\frac{-Q_{b}}{2 \pi} \int_{\pi}^{3 \pi / 2} d \theta \frac{1-\bar{\rho}^{2}}{1+\bar{\rho}^{2}-2 \bar{\rho} \cos (\theta-\phi)}\right] \\
=\frac{-Q_{b}}{2 \pi} \int_{\pi}^{3 \pi / 2} d \theta\left[1+2 \sum_{n=1}^{\infty} \bar{\rho}^{n} \cos (n\{\theta-\phi\})\right], \\
Q_{4}=\frac{-Q_{b}}{2 \pi} \int_{3 \pi / 2}^{2 \pi} d \theta \frac{1-\bar{\rho}^{2}}{1+\bar{\rho}^{2}-2 \bar{\rho} \cos (\theta-\phi)} \\
=\frac{-Q_{b}}{2 \pi} \int_{3 \pi / 2}^{2 \pi} d \theta\left[1+2 \sum_{n=1}^{\infty} \bar{\rho}^{n} \cos (n\{\theta-\phi\})\right] .
\end{aligned}
$$


where $\lambda_{b} L=Q_{b}$ is the beam charge, and $\bar{\rho}=\rho / b$. To perform the integrations in the case of the image charge representation, we make use of known integral identities [4] to obtain

$$
\begin{aligned}
& Q_{1}=-\frac{Q_{b}}{\pi} \arctan \left[\frac{1+\bar{\rho}}{1-\bar{\rho}} \tan \left(\frac{\psi}{2}\right)\right]_{\psi=-\phi}^{\psi=\pi / 2-\phi}, \\
& Q_{2}=-\frac{Q_{b}}{\pi} \arctan \left[\frac{1+\bar{\rho}}{1-\bar{\rho}} \tan \left(\frac{\psi}{2}\right)\right]_{\psi=\pi / 2-\phi}^{\psi=\pi-\phi}, \\
& Q_{3}=-\frac{Q_{b}}{\pi} \arctan \left[\frac{1+\bar{\rho}}{1-\bar{\rho}} \tan \left(\frac{\psi}{2}\right)\right]_{\psi=\pi-\phi}^{\psi=3 \pi / 2-\phi}, \\
& Q_{4}=-\frac{Q_{b}}{\pi} \arctan \left[\frac{1+\bar{\rho}}{1-\bar{\rho}} \tan \left(\frac{\psi}{2}\right)\right]_{\psi=3 \pi / 2-\phi}^{\psi=2 \pi-\phi} .
\end{aligned}
$$

The integrals in the multipole summation representation are also straightforward and result in

$$
\begin{aligned}
& Q_{1}=\frac{-Q_{b}}{2 \pi}\left\{\frac{\pi}{2}+2 \sum_{n=1}^{\infty} \frac{\bar{\rho}^{n}}{n}\left[\sin \left(\frac{n \pi}{4}-\phi_{1}\right)-\sin \left(-\frac{n \pi}{4}-\phi_{1}\right)\right]\right\}, \phi_{1}=\phi-\pi / 4 \\
& Q_{2}=\frac{-Q_{b}}{2 \pi}\left\{\frac{\pi}{2}+2 \sum_{n=1}^{\infty} \frac{\bar{\rho}^{n}}{n}\left[\sin \left(\frac{n \pi}{4}-\phi_{2}\right)-\sin \left(-\frac{n \pi}{4}-\phi_{2}\right)\right]\right\}, \phi_{2}=\phi-3 \pi / 4, \\
& Q_{3}=\frac{-Q_{b}}{2 \pi}\left\{\frac{\pi}{2}+2 \sum_{n=1}^{\infty} \frac{\bar{\rho}^{n}}{n}\left[\sin \left(\frac{n \pi}{4}-\phi_{3}\right)-\sin \left(-\frac{n \pi}{4}-\phi_{3}\right)\right]\right\}, \phi_{3}=\phi-5 \pi / 4 \\
& Q_{4}=\frac{-Q_{b}}{2 \pi}\left\{\frac{\pi}{2}+2 \sum_{n=1}^{\infty} \frac{\bar{\rho}^{n}}{n}\left[\sin \left(\frac{n \pi}{4}-\phi_{4}\right)-\sin \left(-\frac{n \pi}{4}-\phi_{4}\right)\right]\right\}, \phi_{4}=\phi-7 \pi / 4 .
\end{aligned}
$$

In each case, the total charge appearing on the electrode plates can be found to be

$$
Q_{\text {total }}=Q_{1}+Q_{2}+Q_{3}+Q_{4}=-Q_{b}
$$

\section{Finite device length and beam velocity}

Shafer [2] has analyzed the effect of low beam velocities (compared to the speed of light) on the sensitivity and response of capacitive BPMs. His analysis is performed in the frequency domain and applied to beams generated from high frequency (100-800 MHz) RFQ and DTL systems. Shafer identifies several assumptions of the analytic expressions above:

- The BPM electrodes are flush with and grounded to the surface of the conducting beam tube. 
- The beam is a line source (pencil beam).

- The longitudinal extent of the EM field of a beam particle at the beam tube wall is zero, corresponding to a highly relativistic beam.

In the context of high frequency accelerator systems, where the monitored BPM signal is a harmonic of the accelerating frequency, Shafer finds that the effect of low- $\beta$ beam velocity is to reduce the coupling power of high-frequency Fourier components of the beam current to the BPM electrodes while increasing the position sensitivity. In the context of the much lower frequency NDCX-II accelerator, where we have adopted an explicitly time-domain analysis scheme, we expect finite length and time-of-flight effects to introduce temporal smoothing of the intercepted fields. A simple estimate of the time of flight for NDCX-II beam velocities across the 0.5-inch long BPM yields an effective averaging window duration of 2-10 ns. This is equivalent to a low-pass filter that transmits frequencies up to approximately $100 \mathrm{MHz}$.

\section{Circuit model and behavior}

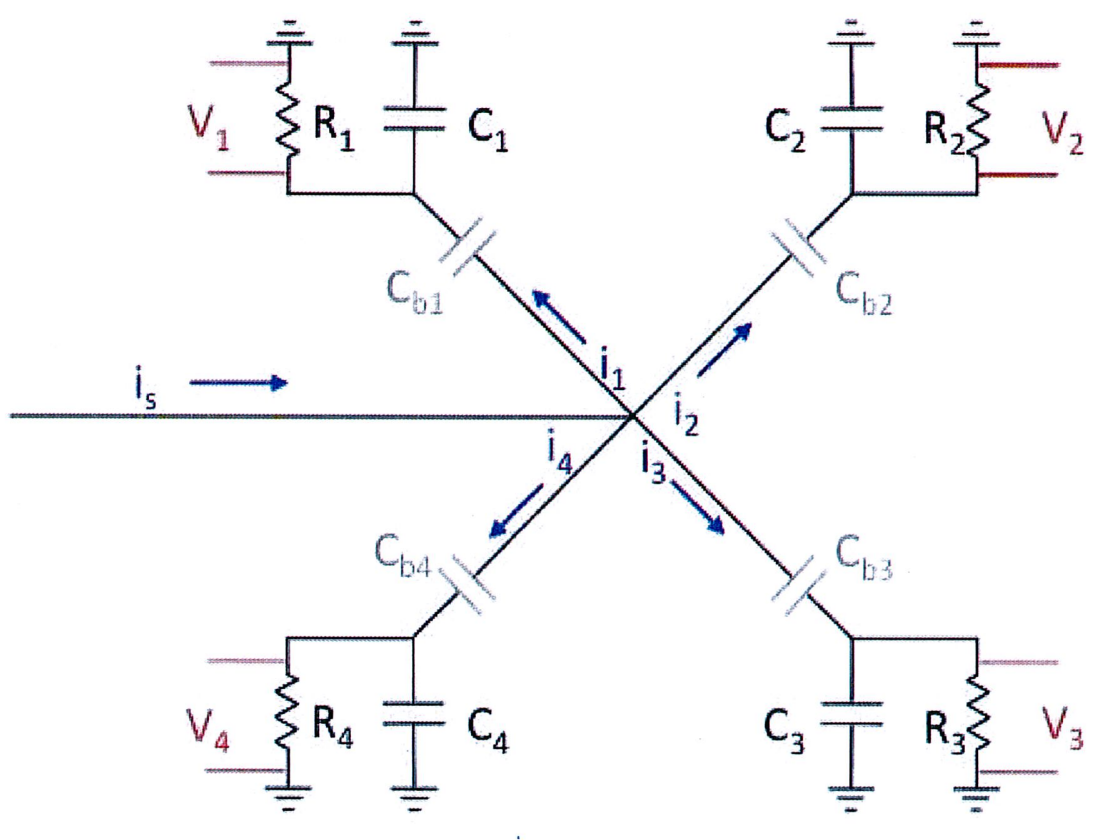

Figure 6: BPM equivalent circuit model 
The behavior and performance of capacitive BPMs are described well in the references $[1,3]$. The symmetric, azimuthally-segmented, four electrode BPM circuit is shown in Figure 6. The incident beam is modeled by the equivalent current element $i_{s}$, which is sub-divided into four currents $\left(i_{1}, i_{2}, i_{3}, i_{4}\right)$ flowing into the separate analyzer circuits. Since the signal detection derives from the capacitive nature of the electrodes in the quasi-static electric field of the beam charge, the equivalent beam current drives from the line charge density, $i_{s}=-d Q_{b} / d t$. Here, the negative sign is introduced to account for the image charge that is intercepted by the electrode plates. The four coupling capacitances $\left(C_{b 1}, C_{b 2}, C_{b 3}, C_{b 4}\right)$ are introduced in an ad hoc form to model the geometry of the beam offset within the beam pipe and the isolation of the physical beam from the analyzing electrodes.

Each BPM analyzer branch is nominally identical. The electrode capacitance to ground is held in parallel to the $50-\Omega$ RG-214 transmission line. The BPM electrode capacitance can be quickly estimated from a coaxial, cylindrical electrode geometry. An inner electrode ring has radius $b$ and the outer electrode ring has radius $b+d$, where $b \sim 4 \mathrm{~cm}$ and $d \sim 1 \mathrm{~mm}$. The dielectric constant of the insulator is $\epsilon / \epsilon_{0} \sim 3.3$. The capacitance per unit length is then $C / l=\frac{2 \pi \epsilon}{\ln \left[\frac{b+d}{b}\right]}(F / m)$. With a 0.5 -inch $(1.27-\mathrm{cm})$ electrode thickness, the ring capacitance is approximately $100 \mathrm{pF}$, or $25 \mathrm{pF}$ per sector electrode.

The actual beam current is related to the beam's line charge density, $\lambda_{b}$, via

$$
\lambda_{b}(t)=\left[\frac{I_{b}(t)}{\beta_{b} c}\right]
$$

where the average beam velocity is denoted by $\beta_{b} c$. The equivalent beam current in the circuit model is the change in induced surface charge per unit time

$$
\begin{aligned}
i_{s} & =-\dot{Q}_{b} \\
& =-L \dot{\lambda_{b}} \\
& =i_{1}+i_{2}+i_{3}+i_{4} .
\end{aligned}
$$

Each branch circuit in the analyzer measures the time-varying electric potential developed across the line impedance. The simple circuit model for each branch leads to a relation between the branch current $\left(i_{n}\right)$ and voltage $\left(V_{n}\right)$ via

$$
i_{n}=C_{n} \dot{V}_{n}+V_{n} / R_{n}
$$

where $C_{n}$ and $R_{n}$ are the coupling capacitance to ground $(25 \mathrm{pF})$ and the line impedance $(50 \Omega)$, respectively. Direct measurement of the line voltage enables reconstruction of the 
branch current and, hence, the induced charges on the electrodes. Relative differences between the induced electrode charges then allow determination of the transverse beam offset position.

\section{Sensitivity and signal levels}

The circuit model, Eqn. 8, can be analyzed for the system sensitivity. For simplicity, assume each branch circuit has identical coupling capacitance and line impedance. Then the total voltage on the line $V_{\text {total }}=V_{1}+V_{2}+V_{3}+V_{4}$ provides a measure of the total current, $i_{s}$, and the line charge density, $\lambda_{b}$. Hence,

$$
\begin{aligned}
i_{s} & =-L \dot{\lambda}_{b} \\
& =C \dot{V}_{\text {total }}+V_{\text {total }} / R
\end{aligned}
$$

or

$$
-L \dot{\lambda_{b}} R=\tau \dot{V}_{\text {total }}+V_{\text {total }}
$$

The time constant $\tau=R C \sim 1 n s$, defines the time-resolution scale of the system.

A simple estimate of the BPM sensitivity is to calculate the response due to a linear ramp in line charge density. Consider a simple ramping time-dependence for the line charge

density from zero to a peak value, $\hat{\lambda_{b}}$, over a rise time, $T_{\text {rise }}$. That is, $\lambda_{b}(t)=t * \hat{\lambda_{b}} / T_{\text {rise }}$. The total voltage response can then be found to be

$$
V_{\text {total }}(t)=-R L \hat{\lambda_{b}} / T_{\text {rise }}[1-\exp (-t / \tau)]
$$

For the case where $T_{\text {rise }} \gg \tau$,

$$
V_{\text {total }}\left(t=T_{\text {rise }}\right) \approx-R L \hat{\lambda_{b}} / T_{\text {rise }}
$$

The nominal sensitivity of the BPM to the peak total line charge density can be estimated to be

$$
\left|V_{\text {total }}\left(t=T_{\text {rise }}\right) / \hat{\lambda_{b}}\right| \approx R L / T_{\text {rise }}
$$

\section{E. Transverse position offset discrimination}

The point of a BPM is to provide an actual measurement of the transverse displacement of the beam's center-of-charge with respect to the electrical center of the BPM itself. 
We first examine the single electrode response from Eqn. 5 in Figure 7. Here the normalized response measures the charge present on the signal electrode plate normalized to the total beam line charge. Figure $7(\mathrm{a})$ shows the normalized response of a single electrode to variations in the position of a point source, with coordinates $(r, \phi)$. At small radial offsets from the axis the response is essentially a single harmonic. At distances much closer to the $\mathrm{BPM}$ electrode radius the response assumes a top-hat distribution in angle. Deviations from the average response (averaged over the full $2 \pi$ angular range) are shown in Figure $7(b)$. By comparing the various individual electrode signals, this will be useful to fiducialize the electrical center of the BPM with respect to the magnetic center of the solenoid and/or the mechanical center of the cell. 


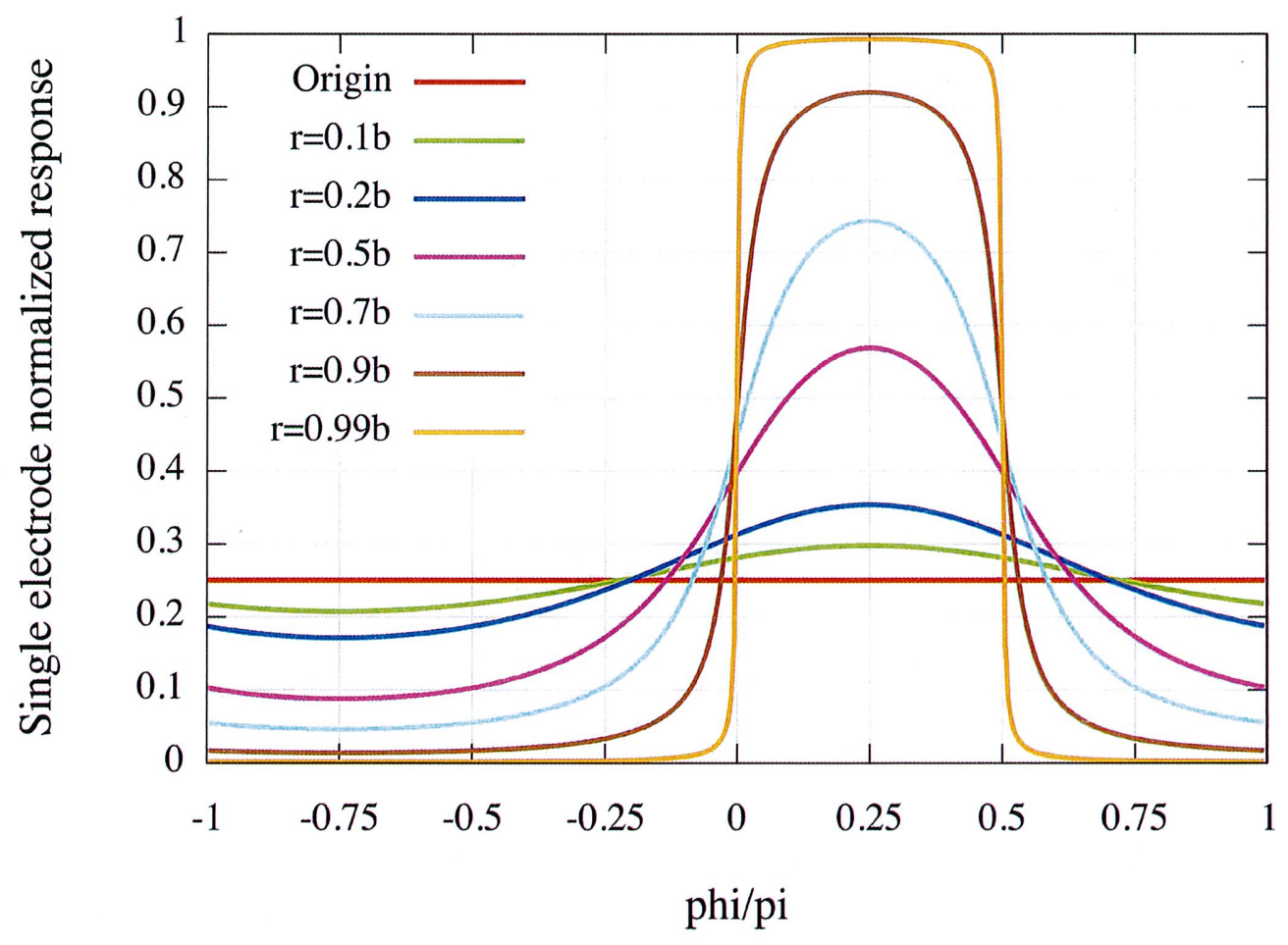

(a)

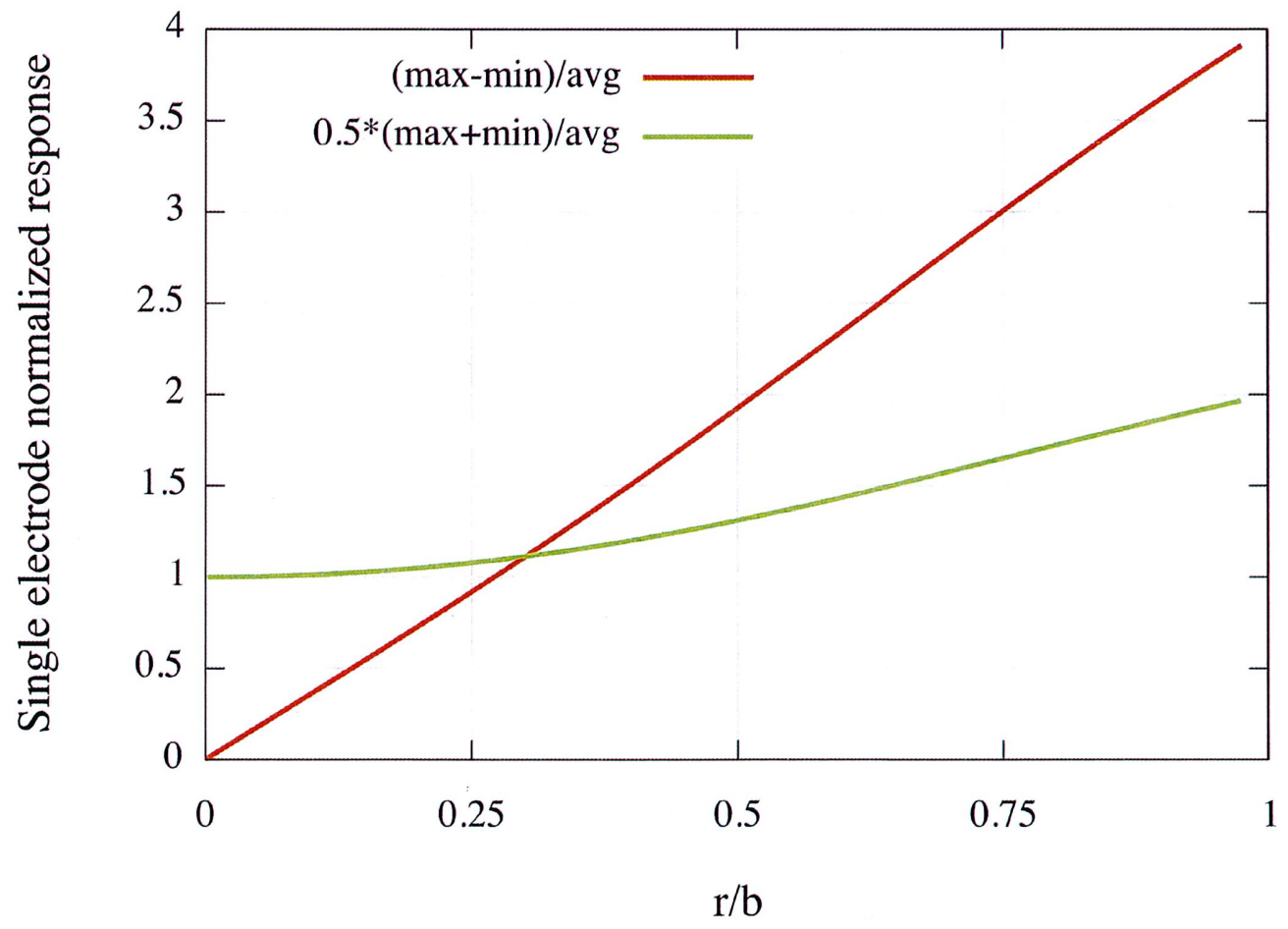

(b)

Figure 7: Single electrode response. 
The equivalent currents flowing into each branch are determined by measuring the potential across the line impedance and solving Eqn. 8. The currents are integrated to find the surface charges appearing on each electrode plate. From Eqn. 6, we relate the beam position and total charge to the charges appearing on individual electrodes. We typically assume that the beam's center-of-charge makes only a small offset from the BPM electrical axis with respect to the BPM electrode radius (which is nominally equal to the beam pipe radius). In this case, we can retain only the first term $(n=1)$ in the series in Eqn. 6 with small loss of accuracy

$$
\begin{aligned}
& Q_{1} \approx \frac{-Q_{b}}{2 \pi}\left\{\frac{\pi}{2}+2 \bar{\rho}[\cos \phi+\sin \phi]\right\}=\frac{-Q_{b}}{2 \pi}\left\{\frac{\pi}{2}+2[\delta \bar{x}+\delta \bar{y}]\right\} \\
& Q_{2} \approx \frac{-Q_{b}}{2 \pi}\left\{\frac{\pi}{2}+2 \bar{\rho}[\sin \phi-\cos \phi]\right\}=\frac{-Q_{b}}{2 \pi}\left\{\frac{\pi}{2}+2[-\delta \bar{x}+\delta \bar{y}]\right\}, \\
& Q_{3} \approx \frac{-Q_{b}}{2 \pi}\left\{\frac{\pi}{2}+2 \bar{\rho}[-\cos \phi-\sin \phi]\right\}=\frac{-Q_{b}}{2 \pi}\left\{\frac{\pi}{2}+2[-\delta \bar{x}-\delta \bar{y}]\right\} \\
& Q_{4} \approx \frac{-Q_{b}}{2 \pi}\left\{\frac{\pi}{2}+2 \bar{\rho}[-\sin \phi+\cos \phi]\right\}=\frac{-Q_{b}}{2 \pi}\left\{\frac{\pi}{2}+2[\delta \bar{x}-\delta \bar{y}]\right\} .
\end{aligned}
$$

To calculate the beam offset we take symmetric differences and normalize to the sum

$$
\begin{aligned}
& \delta x=b \delta \bar{x}=b \frac{\left(Q_{1}+Q_{4}\right)-\left(Q_{2}+Q_{3}\right)}{\left(Q_{1}+Q_{2}+Q_{3}+Q_{4}\right)} \\
& \delta y=b \delta \bar{y}=b \frac{\left(Q_{1}+Q_{2}\right)-\left(Q_{3}+Q_{4}\right)}{\left(Q_{1}+Q_{2}+Q_{3}+Q_{4}\right)} .
\end{aligned}
$$




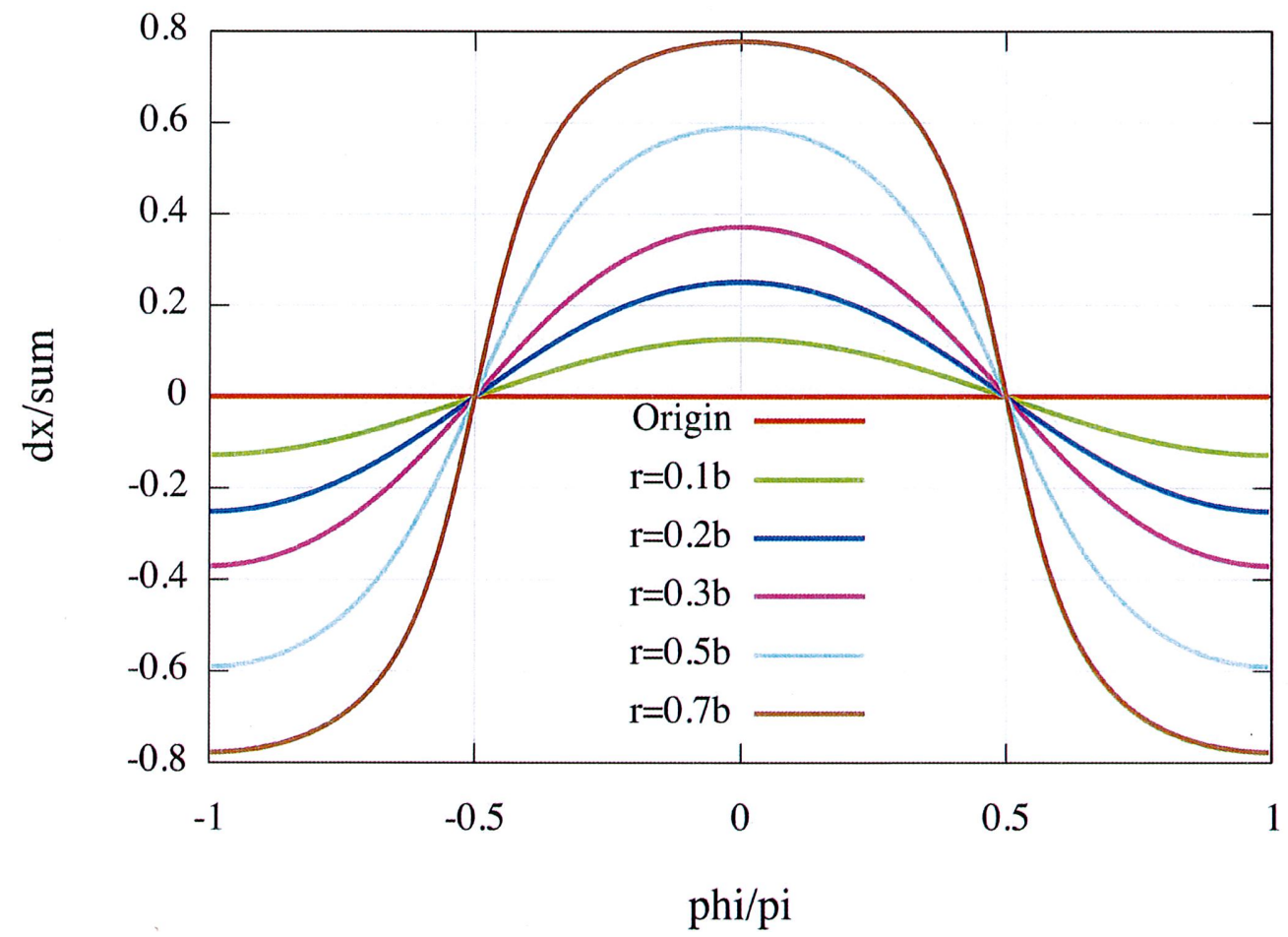

(a)

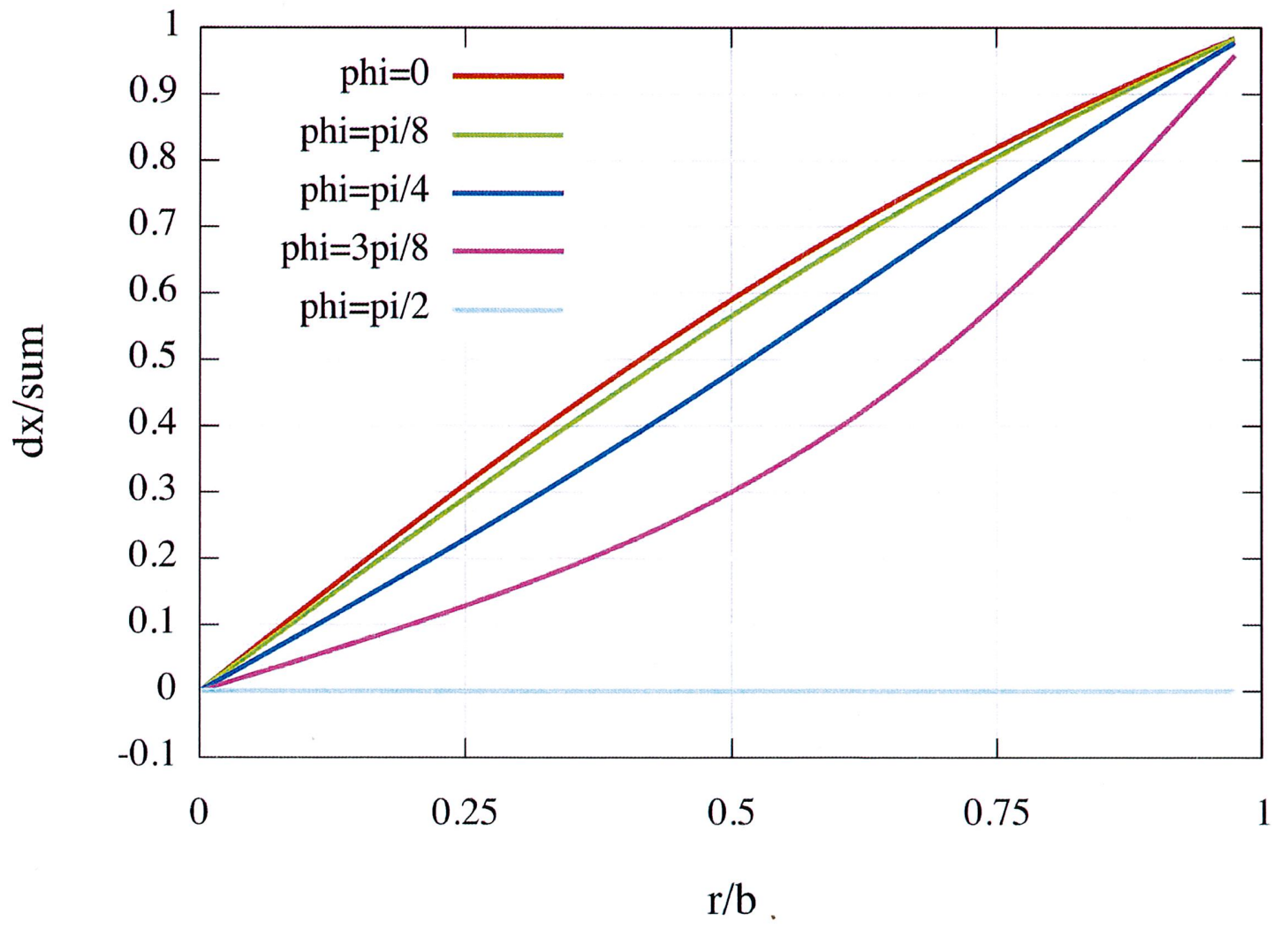

(b)

Figure 8: Theoretical BPM response 
The normalized electrode differences $\frac{\left(Q_{1}+Q_{4}\right)-\left(Q_{2}+Q_{3}\right)}{\left(Q_{1}+Q_{2}+Q_{3}+Q_{4}\right)}$ obtained from Eqn. $\overline{5}$ are shown in Figure 8. We can compare the response shown in Figure 8(a) to a harmonic series representation and obtain an estimate of the error resulting in our assumption leading to Eqn. 13. The harmonic series representation (using the symmetry in Figure 8(a)) follows as

$$
\delta \bar{x}=\frac{\left(Q_{1}+Q_{4}\right)-\left(Q_{2}+Q_{3}\right)}{\left(Q_{1}+Q_{2}+Q_{3}+Q_{4}\right)}=\sum_{n=1,3,5,7, \ldots} a_{n} \cos (n \phi) .
$$

A nonlinear fitting routine is used to determine the coefficients in the series for the various radial offset cases considered. These coefficients are tabulated in Table I below. Linearity is retained with error $<5 \%$ for radial offsets $r / b<0.4$.

\begin{tabular}{|c|c|c|c|c|c|c|}
\hline $\mathrm{r} / \mathrm{b}$ & $a_{1}$ & $a_{3}$ & $a_{5}$ & $a_{7}$ & $a_{9}$ & Deviation from linearity (\%) \\
\hline \hline 0.0 & 0 & 0 & 0 & 0 & 0 & 0.0 \\
\hline 0.1 & 0.127 & 0 & 0 & 0 & 0 & 0.0 \\
\hline 0.2 & 0.255 & -0.003 & 0 & 0 & 0 & 1.2 \\
\hline 0.3 & 0.382 & -0.011 & 0.001 & 0 & 0 & 2.9 \\
\hline 0.4 & 0.509 & -0.027 & 0.003 & 0 & 0 & 5.3 \\
\hline 0.5 & 0.637 & -0.053 & 0.008 & -0.001 & 0 & 8.4 \\
\hline 0.6 & 0.764 & -0.092 & 0.020 & -0.005 & 0.001 & $>12$. \\
\hline 0.7 & 0.891 & -0.146 & 0.043 & -0.015 & 0.006 & $>17$. \\
\hline 0.8 & 1.019 & -0.217 & 0.083 & -0.038 & 0.019 & $>23$. \\
\hline 0.9 & 1.146 & -0.309 & 0.150 & -0.087 & 0.055 & $>31$. \\
\hline
\end{tabular}

Table I: Harmonic series coefficients. 


\section{TEST MEASUREMENTS}

A. Signal level and coupling characterization

Test stand layout and instrumentation

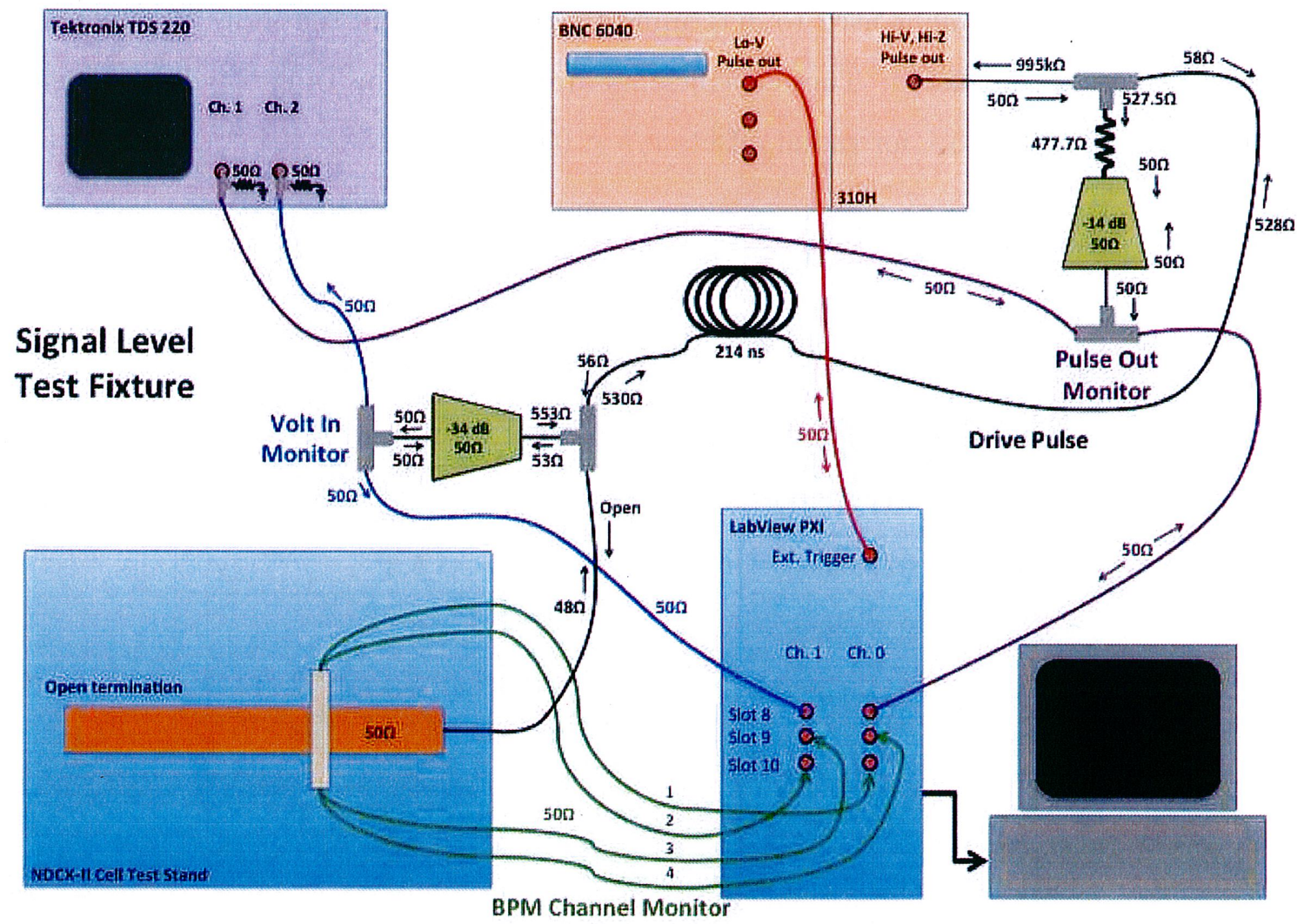

Figure 9: Test stand for signal level calibration

The test stand to measure signal coupling is shown schematically in Figure 9. A high voltage $(0-500 \mathrm{~V})$, short pulse $(<300 \mathrm{~ns})$ train originates from the BNC 6040 Universal Pulse Generator and BNC $310 \mathrm{H}$ high-voltage module. The generator output is monitored in the Pulse Out monitor circuit. A delay line is introduced between the generator and the load to eliminate the overlap of primary pulses with reflected pulses. Following the delay line, a Volt In monitor is used to calibrate the waveform of the pulse entering the test device.

The test device itself is an NDCX-II accelerator cell with fore and aft vacuum flanges. 
A BPM has been attached to the ground plane of the cell. A solid $13 / 8$-inch diameter, 35-inch long copper rod is inserted along the accelerator and BPM axis. A 3 1/8-inch inner diameter stainless steel tube is mounted at the input flange end, coaxial to the rod axis. A 1-cm longitudinal gap exists between the BPM and the stainless steel tube. The rod and tube, along with the accelerator cell, now define by geometry a $\check{\jmath} 0-\Omega$ coaxial line. The downstream end of the coaxial line is left un-terminated in an open-circuit configuration. The input flange accepts the $50-\Omega$ line from the generator and passes it to the load without an impedance mismatch. The output cables from the BPM are $50-\Omega$ RG-214 and are also terminated in feedthroughs at the test device input vacuum flange. The signal cables are terminated into $50 \Omega$ at the National Instruments NI PXI-104う chassis with NI PXI-ð̃ high-bandwidth digitizer cards.

Besides the individual BPM electrode signals, the digitizer chassis also accepts output from both the Pulse Out and Volt In monitor circuits. These two circuits are also monitored on a nearby Tektronix TDS 220 digital sampling scope. The low voltage $(4 \mathrm{~V})$ output of the BNC 6040 generator is tapped to provide a trigger signal for the NI PXI chassis. 
Pulser and analyzer circuit calibrations

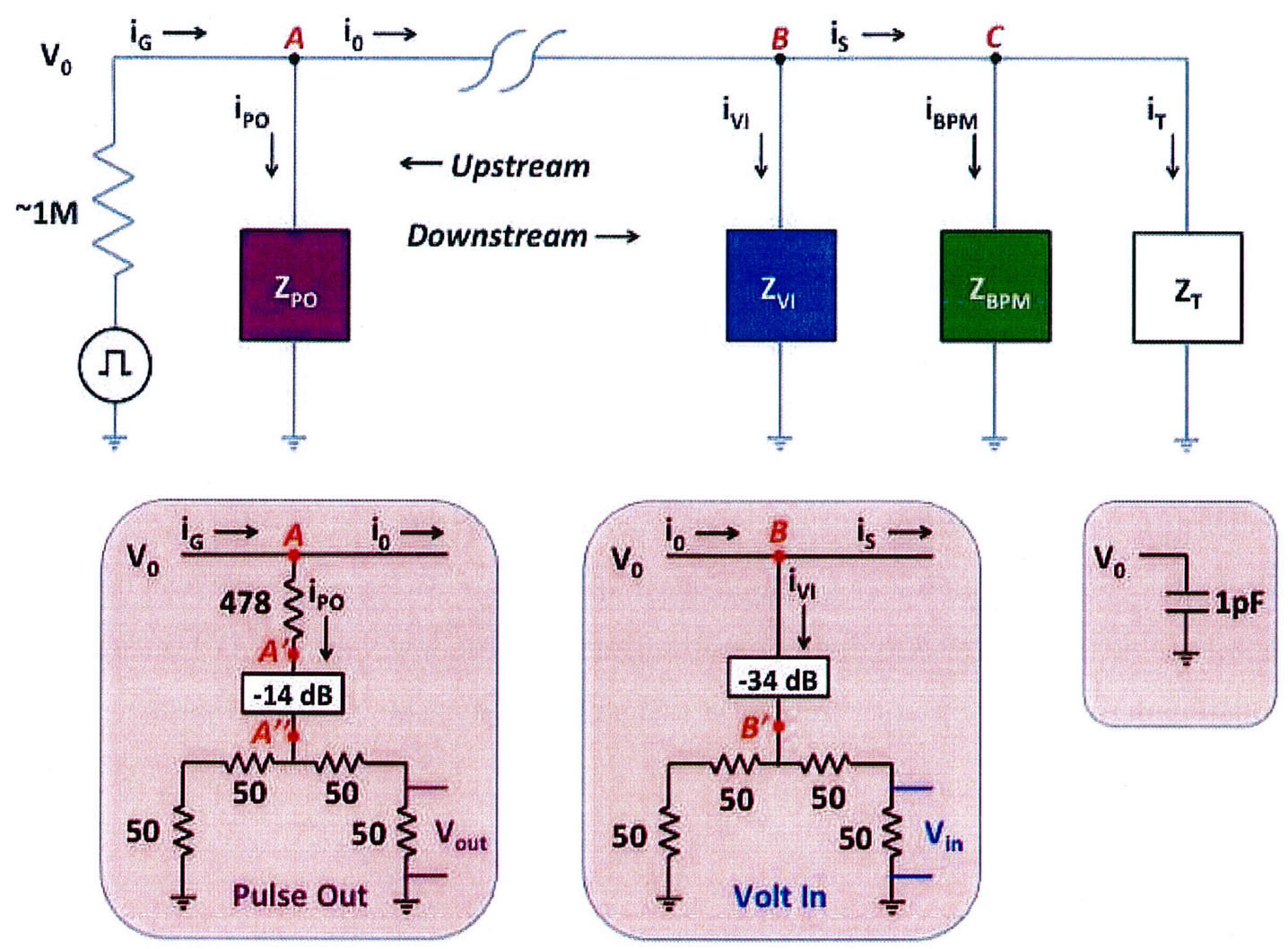

Figure 10: Signal level test circuits.

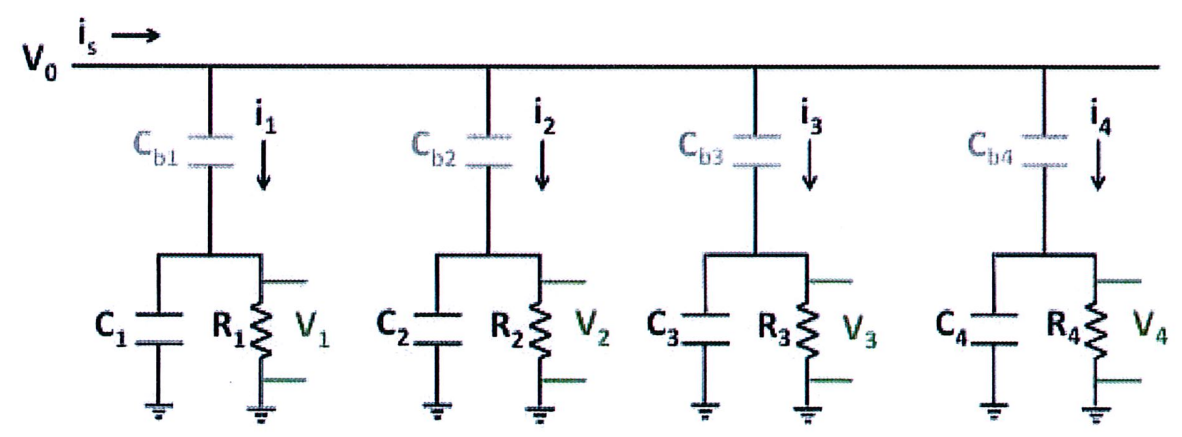

Figure 11: BPM analyzer branch circuits. 
The monitoring circuits in the test fixture are shown schematically in Figure 10. The BPM analyzer branch circuits are shown in Figure 11. Indicated in the figure are the upstream and downstream directions of signal flow which translate to towards or away from the generator, respectively. The Pulse Out and Volt In monitor circuits are shown in detail insets.

\begin{tabular}{|c|c|c|c|}
\hline Observation Point & Lpstream $(\Omega)$ & Downstream $(\Omega)$ & Through Monitor $(\Omega)$ \\
\hline $\mathrm{A}$ & $99 \check{~ k ~}$ & 57 & $う 30$ \\
\hline$A^{\prime}$ & - & - & 52 \\
\hline$A^{\prime \prime}$ & - & - & 50 \\
\hline B & 530 & $\infty$ & 53 \\
\hline $\mathrm{B}^{\prime}$ & - & - & 50 \\
\hline
\end{tabular}

Table II: Measured impedances in signal level test circuits.

Particular observation points in the drive and observation circuits are indicated in Figure 10. The line impedances at these points have been measured and are tabulated in Table II. These measured impedance values are used to derive the essential calibrations at the monitoring stations:

$$
\begin{aligned}
V_{0}(V) & =101.9 * V_{\text {out }}(V), \\
i_{0}(A) & =93 / 50 * V_{\text {out }}(V), \\
V_{0}(V) & =100 * V_{\text {in }}(V), \\
i_{s}(A) & =53 / 25 * V_{\text {in }}(V) .
\end{aligned}
$$

The BPM analyzer branch circuits are designed to be identical, with $R_{1}=R_{2}=R_{3}=$ $R_{4}=R=50 \Omega$ and $C_{1}=C_{2}=C_{3}=C_{4}=C=25 \mathrm{pF}$. The coupling capacitances $\left(C_{b 1}, C_{b 2}, C_{b 3}, C_{b 4}\right)$ in the test fixture are defined by the capacitance per unit length of the coaxial line defined by the beam pipe and BPM as the outer conductor and the copper rod as the center conductor. Coaxial symmetry of the line dictates the equality of the coupling capacitances. A simple calculation reveals $C_{b 1}=C_{b 2}=C_{b 3}=C_{b 4}=C_{b} \sim 0.2 p F$. The potential drop across this coupling capacitance in each branch is denoted then by $V_{b}$. The line voltage, $V_{0}$, is then determined from

$$
V_{0}=V_{b}+V_{1}=V_{b}+V_{2}=V_{b}+V_{3}=V_{b}+V_{4}
$$


The relationship between observed branch line potentials and the currents flowing in each individual branch is given in Eqn. 8. The total line current density entering the BPM is given from Eqn. 7, and the line potential is then calculated using the calibrations in Eqns. 16.

\section{Measurements and Results}

Signal level measurements were performed by generating $1-\mathrm{Hz}$ pulse trains from the BNC 6040 generator, with set pulse durations and peak amplitude. A LabView routine was utilized to acquire the voltage waveforms from the Pulse Out, Volt In, and the BPM electrode monitoring circuits. 20 shots were acquired and archived, and this was repeated for various combinations of pulse duration and peak amplitude. The signals were analyzed offline with the help of a specialized Python routine.

The analysis routine, as utilized here, performs a sequence of operations on a multi-shot set of waveforms: (i) each waveform is processed to remove any baseline bias; (ii) an average waveform is calculated from a specified number of independent shots (20 shots in these examples); (iii) a Gaussian window time-average is performed to remove high frequency noise components and to model finite device length and time-of-flight (100 $\mathrm{MHz}$ band pass is utilized here); (iv) finally, a 3rd order spline representation of the waveform is constructed to improve the degree of continuity in numerically calculated time derivatives.

Figures 12 and 13 display the results from two of the runs. Shown are the BPM monitor signals; the line voltage $\left(V_{0}\right)$ derived from the three sets of monitoring circuits; and finally, the line charge density derived from the Volt In and the BPM monitoring circuits. The capacitive coupling nature of the BPM electrode is evident in the waveform signals. The pulse line voltage is seen to attenuate by $10 \%$ between the generator output and the Volt In monitor. The line voltage determined by analyzing the BPM signals is correct to within $5 \%$ error. The line charge density is determined by a scaling factor, and shows the same level of agreement between the Volt In and the BPM monitors. 

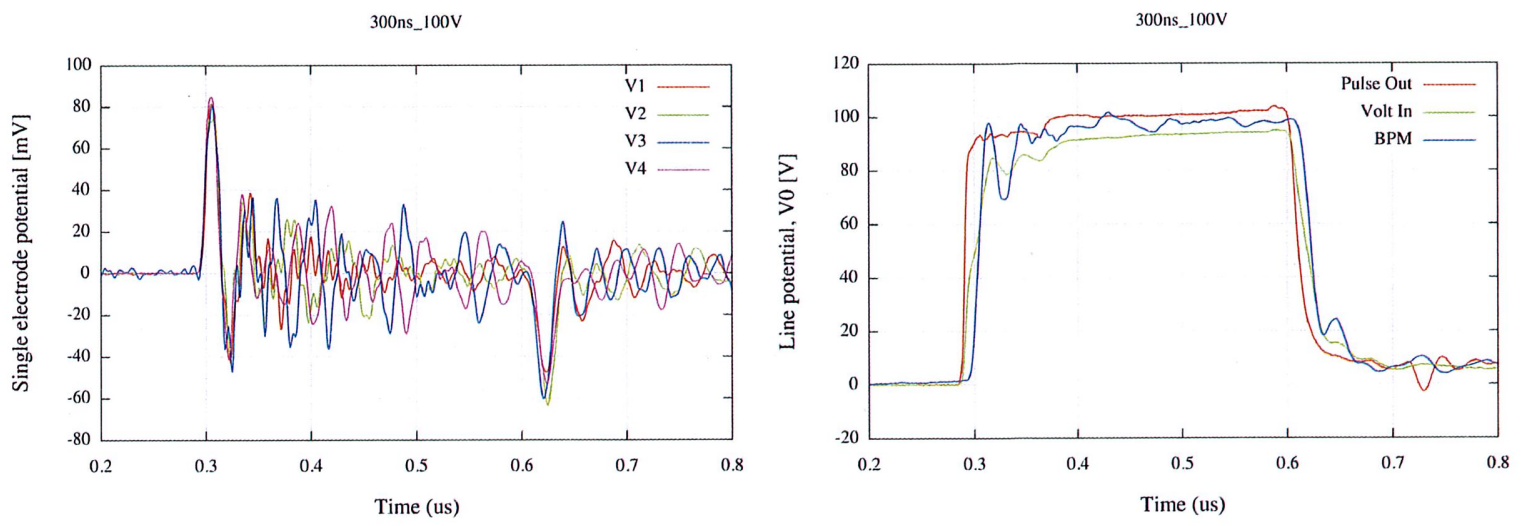

(a) Electrode monitor potentials (left), and derived line potential (right).

300ns_100V

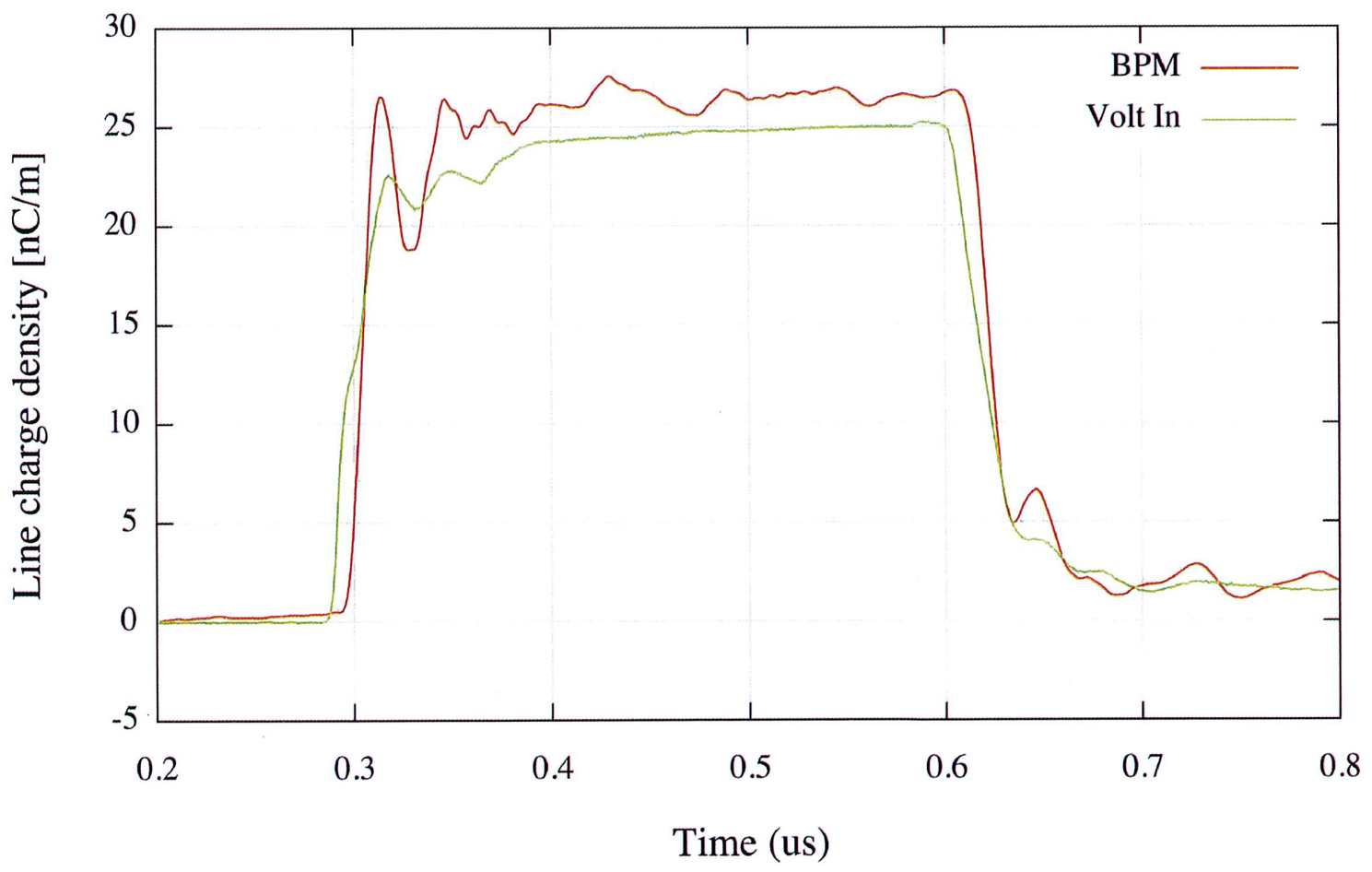

(b) Calculated line charge density, $\lambda_{b}$.

Figure 12: BPM response to 300ns, 100V drive pulse. 

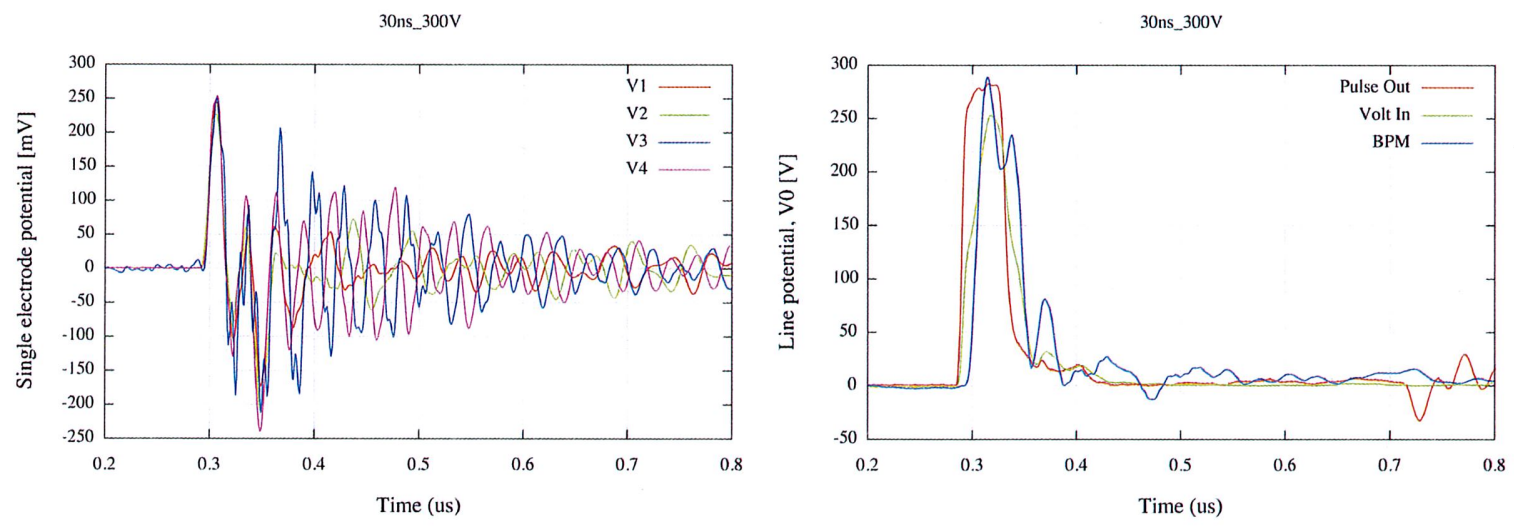

(a) Electrode monitor potentials (left), and derived line potential (right).

30ns_300V

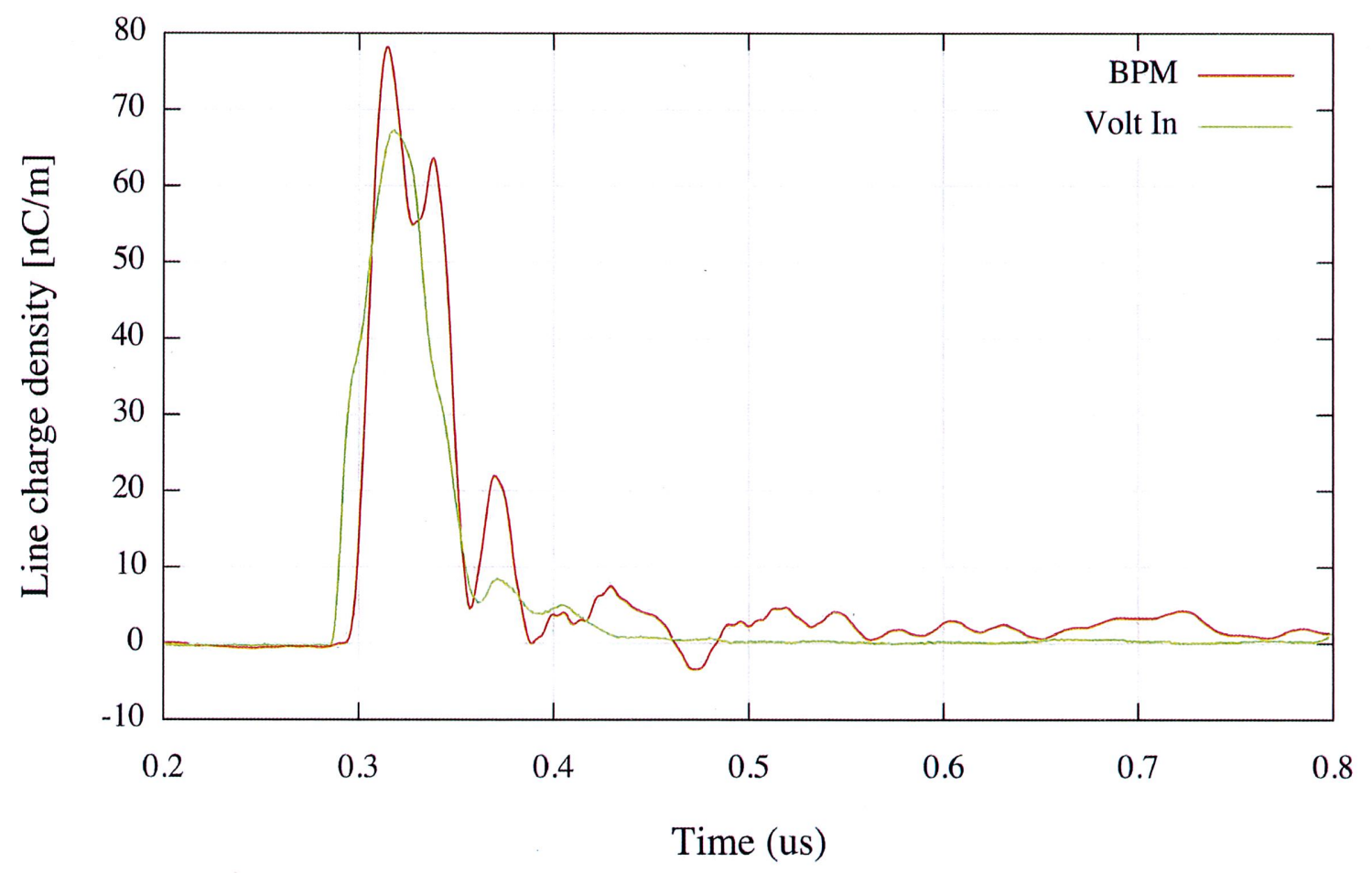

(b) Calculated line charge density, $\lambda_{b}$.

Figure 13: BPM response to 30ns, 300V drive pulse. 


\section{Sensitivity Scaling}

Compiled from a series of runs of the various cases of pulse duration and peak amplitude, we construct a comparison in Table III. We compare the sensitivity results in the Table to the rough estimate provided by Eqn. 12. Assuming a pulse rise-time of approximately $19 \mathrm{~ns}$, a transmission line impedance of $50 \Omega$, and an electrode length of $1.27 \mathrm{~cm}$, the estimated sensitivity is $33 \mathrm{mV} / \mathrm{nC} / \mathrm{m}$ for all electrodes together, or $8.3 \mathrm{mV} / \mathrm{nC} / \mathrm{m}$ for an individual electrode. This estimate is seen to agree with measured results to better than $20 \%$ over a wide range of pulse parameters. The main error in the estimate lies with the assumption of linearity in the pulse rising edge and of the pulse rise time being much shorter in duration than the pulse flattop. This degree of sensitivity should be sufficient to measure variations in line charge density at the $1 \mathrm{nC} / \mathrm{m}$ level, or at finer scales with appropriate signal amplification.

\begin{tabular}{|c|c|c|c|c|c|}
\hline Pulse duration & Peak $V_{0}$ & Peak $V_{0}$, & Peak single electrode & Peak $\lambda_{b}$ & $V_{\text {electrode }} / \lambda_{b}$ \\
\hline \hline$(\mathrm{ns})$ & Pulse Out $(\mathrm{V})$ & Volt In \& BPM $(\mathrm{V})$ & potential, $V_{\text {electrode }(\mathrm{mV})}$ & $(\mathrm{nC} / \mathrm{m})$ & $(\mathrm{mV} / \mathrm{nC} / \mathrm{m})$ \\
\hline \hline 30 & 46 & 40 & $2 \check{1}$ & 11 & 9.1 \\
\hline 30 & 95 & 80 & 50 & 22 & 9.1 \\
\hline 30 & $18 \check{5}$ & 160 & 100 & 45 & 8.9 \\
\hline 30 & 280 & 250 & 150 & 68 & 8.8 \\
\hline 100 & 17.5 & 16.5 & 10 & 4.5 & 8.9 \\
\hline 100 & 45 & 42 & 25 & 11 & 9.1 \\
\hline 100 & 92 & 84 & 50 & 23 & 8.7 \\
\hline 300 & 8.5 & 8 & 5 & 2 & 10.0 \\
\hline 300 & 18.5 & 17 & 10 & 4.8 & 8.3 \\
\hline 300 & 49 & 45 & 25 & 12 & 8.3 \\
\hline 300 & 100 & 90 & 50 & 25 & 8.0 \\
\hline
\end{tabular}

Table III: Scaling of line charge density, pulse duration, and sensitivity. 


\section{B. Electrode characterization and position sensitivity}

Test stand layout and instrumentation

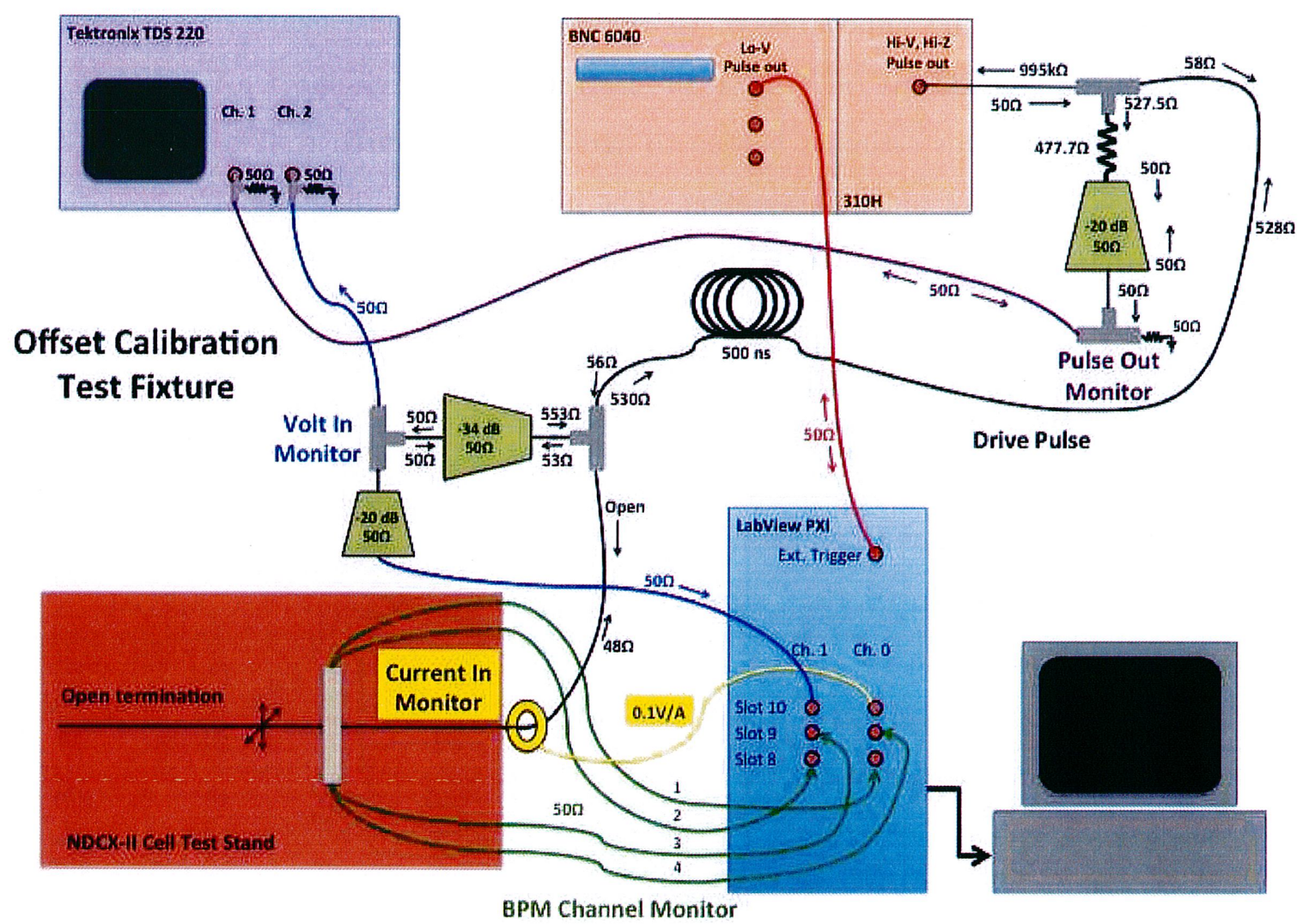

Figure 14: Test circuit for displacement calibration

A separate facility to measure signals from transversely offset, pulsed currents was installed within the NDCX-II magnet alignment test stand. The high voltage pulser, analyzing circuits, and data acquisition system used in the previous set of BPM signal level measurements were stationed to generate a current pulse along the transversely-positionable stretched wire in the magnet test facility. The wire was positioned via LabView controls. The measurement test stand is shown schematically in Figure 14. The need for a positionable wire complicates the circuit used to pulse the wire. In particular, the pulsed wire line impedance can not be maintained at a near-constant $50 \Omega$ over the several meter length between the positioning towers. As a result, reflections will occur that complicate the anal- 
ysis. We lose the ability to make direct comparisons between the waveforms obtained at the Pulse Out monitor and the monitors in close proximity to the BPM itself.

Pulser and analyzer circuit calibrations

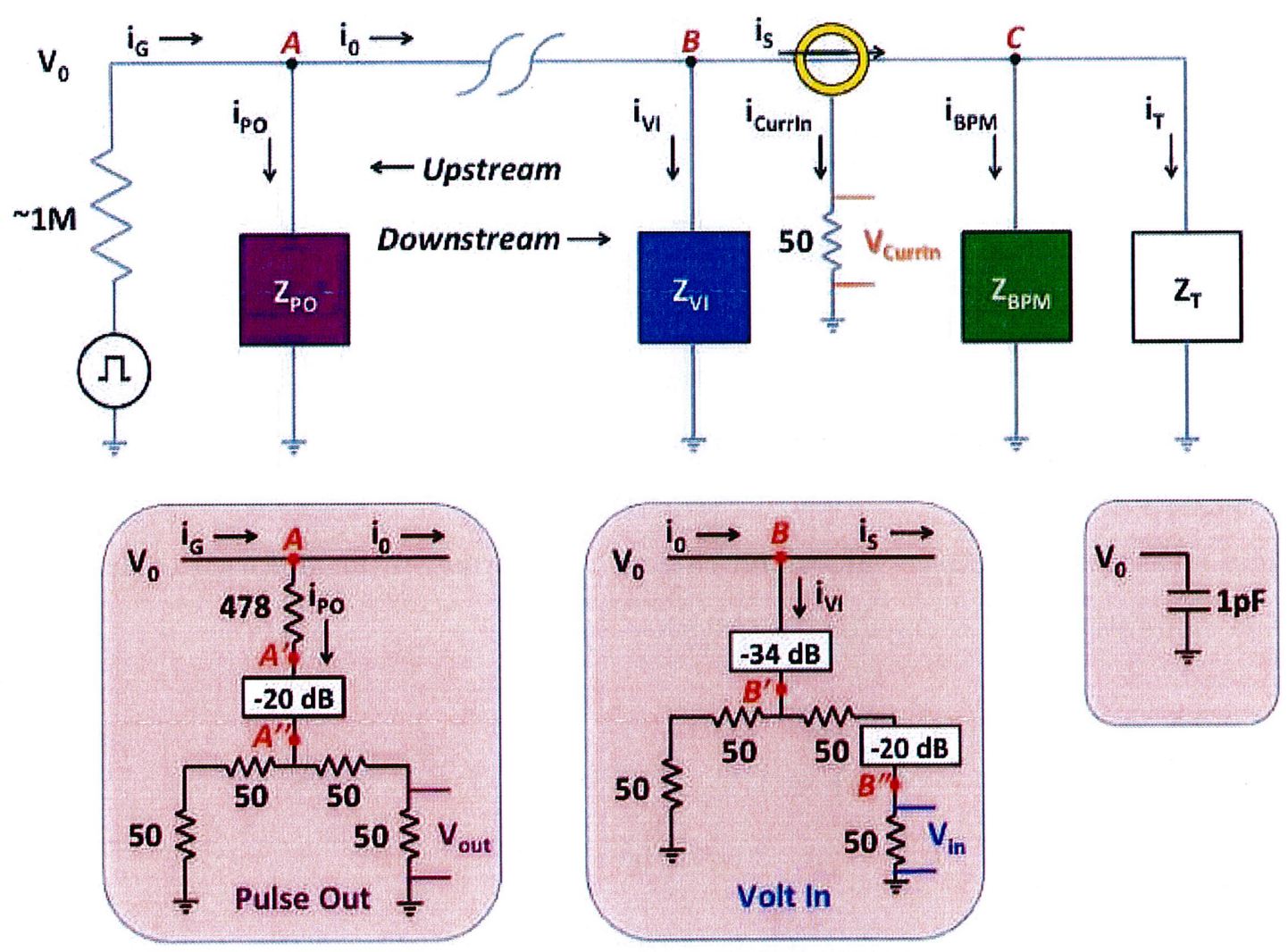

Figure 15: Displacement test circuits.

The monitoring circuits in the test fixture are shown schematically in Figure 15. The BPM analyzer branch circuits are shown in Figure 11. Indicated in the figure are the upstream and downstream directions of signal flow which translate to towards or away from the generator, respectively. The Pulse Out and Volt In monitor circuits are shown in detail insets. 


\begin{tabular}{|c|c|c|c|}
\hline Observation Point & Lpstream $(\Omega)$ & Downstream $(\Omega)$ & Through Monitor $(\Omega)$ \\
\hline \hline A & $995 \mathrm{k}$ & 57 & 530 \\
\hline A' & - & - & 52 \\
\hline A" & - & - & 50 \\
\hline B & 530 & $\infty$ & 53 \\
\hline B' & - & - & 50 \\
\hline B" & - & - & 50 \\
\hline
\end{tabular}

Table IV: Measured impedances in displacement test circuits.

Particular observation points in the drive and observation circuits are indicated in Figure 15. The line impedances at these points have been measured and are tabulated in Table IV. A separate current monitor (Ion Physics Corporation CM-10-L) has been installed to measure the current flowing in the line observed at the entrance positioning tower just upstream from the BPM.

These measured impedance values and current transformer ratios are used to derive the essential calibrations at the monitoring stations:

$$
\begin{aligned}
& i_{s}(A)=V_{\text {CurrIn }}(V) /(0.1 V / A), \\
& i_{s}(A)=20 * 53 / 50 * V_{i n}(V) .
\end{aligned}
$$

As before, the relationship between observed BPM branch line potentials and the currents flowing in each individual branch is given in Eqn. 8. The total line current density entering the BPM is given from Eqn. 7, and the line potential is then calculated using the calibrations in Eqns. 18. To measure absolute beam offsets, we make use of Eqn. 14. This analysis requires only knowledge of the BPM electrode radius, and the difference-over-sum of the charges appearing on the BPM branch electrode plates, Eqn. 14.

\section{Offset Measurement and Calibration}

Variations to transverse beam offset in the individual electrode responses were measured verify the BPM functionality and calibration. Using the test stand described above (see 
Figure 14) a thin wire was placed at an arbitrary fiducial position (the "origin"), as well as 8-12 equally spaced azimuthal positions at a constant radius about this origin. At each position (including the origin), pulses were generated along the wire and the BPM electrode signals were recorded. The signals from the upstream current monitor as well as the Volt In monitor circuit were also recorded. The output pulses from the generator had an amplitude of $500 \mathrm{~V}$ and $30 \mathrm{~ns}$ duration (FWHM).

The waveforms detected by the BPM electrode monitoring circuits and the current monitor are shown in Figure 16(a) below. Analyzing the individual waveforms, we obtain the beam line charge density at the BPM position in Figure 16(b). These waveforms are measured at the nominal wire fiducial offset position ("origin").

Additional waveform sets are collected as the pulsed wire is repositioned at various locations that span $2 \pi$ radians of azimuth at constant radius from the nominal fiducial position. The waveforms are again analyzed to obtain the time history of the line charge density induced on the electrodes. In Figure 17(a) we show the variation of the electrode line charge density averaged over $\check{5} 0 \mathrm{~ns}$ as a function of azimuthal position. The data is shown as discrete points, and the variation with position is then fit (in a least squares sense) to a single harmonic variation (Eqn.15 with $n=1$ ) and plotted with a solid line. The wire offsets are calculated from Eqn. 14 for both the measured points as well as the fitted ones and are plotted in Figure 17(b).

A future note will examine BPM response and measurement sensitivity and accuracy. In particular, we will study the effects of signal-to-noise, waveform averaging, shot-to-shot variations and post-processing analysis on derived offset accuracy and precision. Another note will examine the sensitivity and response of the BPM monitoring system to the various baseline NDCX-II operating scenarios. 
18mmOffset_8mmRadius

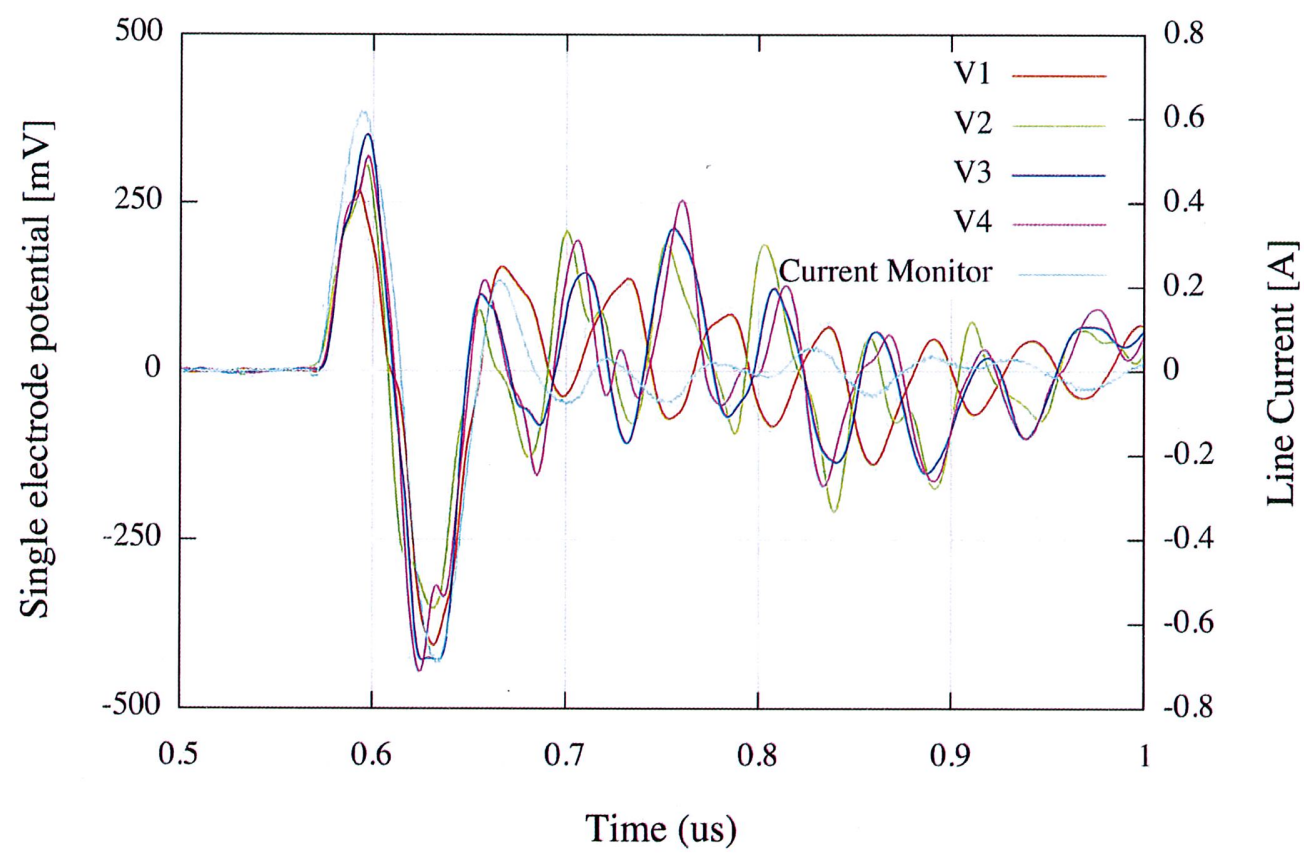

(a)

$18 \mathrm{mmOffset}$ 8mmRadius

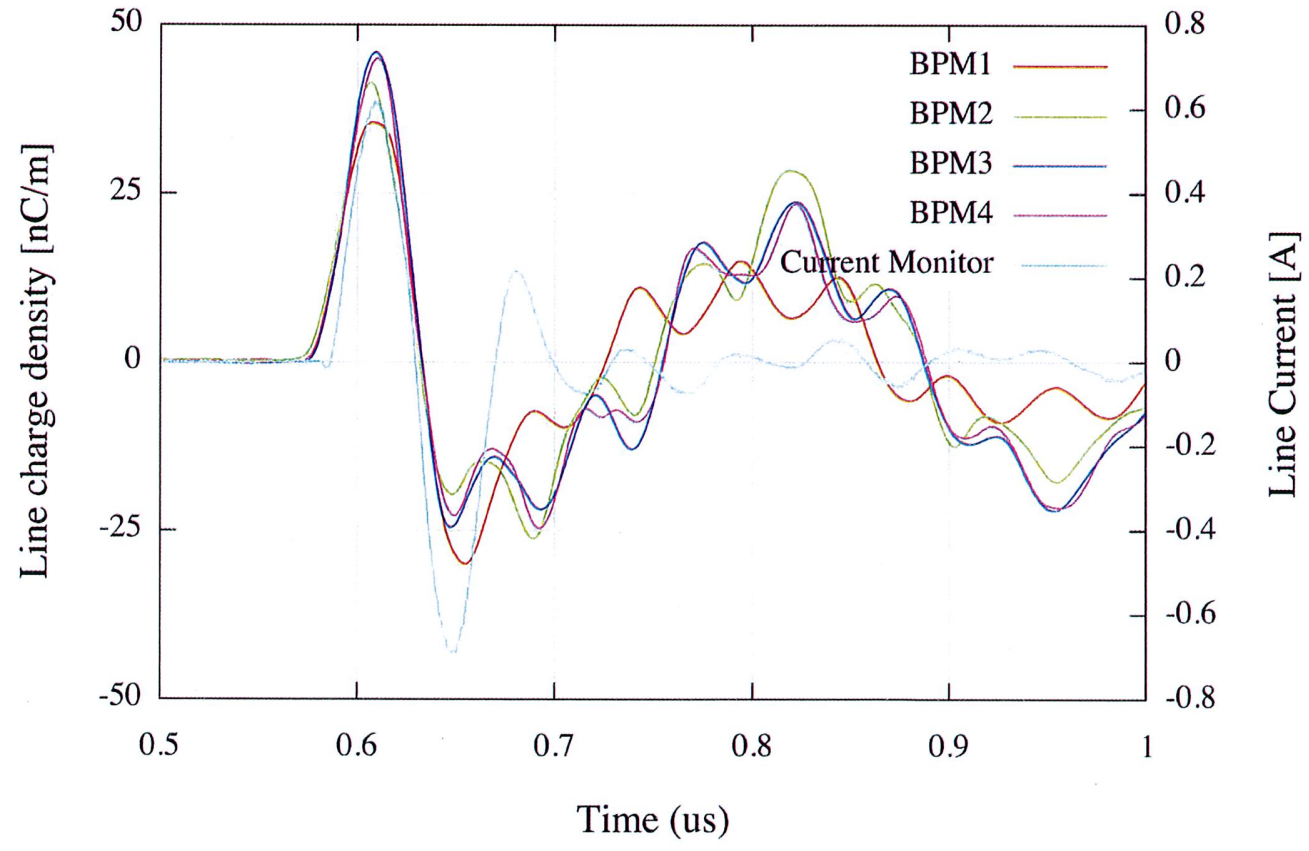

(b)

Figure 16: BPM electrode potential (a), and line charge density (b). 
18mmOffset_8mmRadius, 0.57:0.62 usec

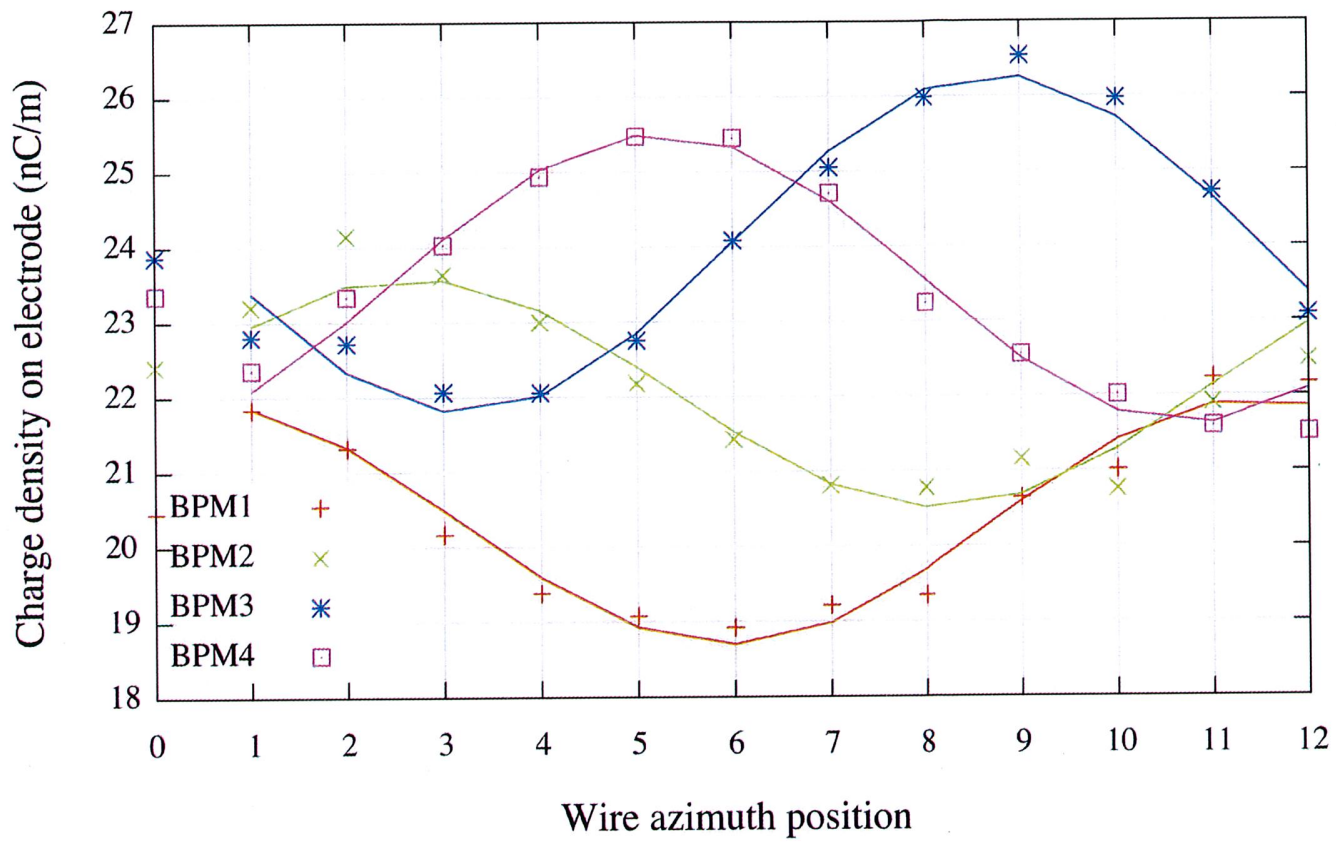

(a)

18mmOffset_8mmRadius, 0.57:0.62 usec

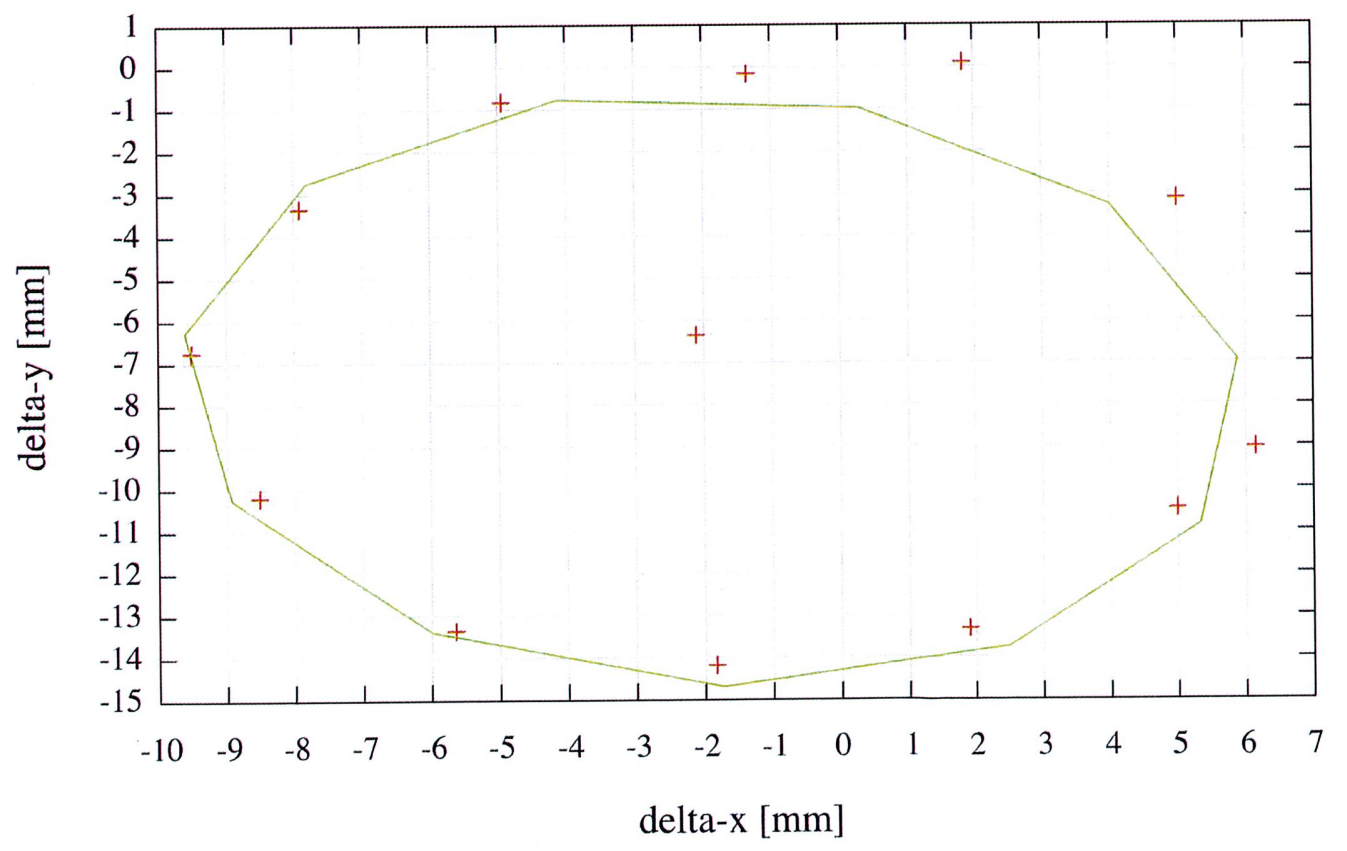

(b)

Figure 17: BPM signals and offset measurements from displacement. 


\section{Tuning Algorithms}

We use the previously described procedure to calibrate and align individual BPMs to the rest of the beamline. Here, we will align the BPM with respect to the magnetic beamline of the lattice. Each accelerator cell structure incorporates externally accessible fiducial markers that reference the mechanical beamline center, and are used to reference relative and absolute position. Solenoid magnets are measured, shimmed mechanically, and installed in each cell. On the magnet test stand, each cell is mounted and the magnetic center line is measured by the pulsed wire technique. Deviations from the mechanical center line are recorded.

A BPM is mounted to the ground plane flange of an upstream (to the diagnostic cell, that is) accelerator cell. A pulsed wire measurement establishes the magnetic center line of the solenoid magnet. This position is used as the fiducial origin for the BPM calibration. Repeating the process of sweeping the pulsed wire about the azimuth at a predetermined radius, we measure and calibrate the response of the BPM unit assigned to that cell.

\section{Acknowledgments}

We acknowledge the skilled technical assistance of Thomas Lipton, Ahmet Pekedis, Craig Rogers, and Will Waldron.

[1] R. Shafer, "Beam Position Monitoring", AIP Conference Proceedings 212 (1990), pp. 26-う̌.

[2] R. Shafer, "Beam Position Monitor Sensitivity for Low- $\beta$ Beams", AIP Conference Proceedings 319 (1994), pp. 303-308.

[3] P. Strehl, "Beam Instrumentation and Diagnostics", Springer (2006).

[4] I.S. Gradshteyn and I.M. Ryzhik, "Tables of Integrals, Series, and Products, כth Ed.", Academic Press, Inc. (1994), Eqn. 2.5̌́6. 